\title{
On the stability of nonconservative continuous systems under kinematic constraints
}

\author{
J. Lerbet ${ }^{1, *}$, N. Challamel ${ }^{2}$, F. Nicot ${ }^{3}$, and F. Darve ${ }^{4}$ \\ ${ }^{1}$ IBISC, UFRST-UEVE, 40, rue du Pelvoux CE 145591020 Evry Courcouronnes cedex, France \\ ${ }^{2}$ Université Européenne de Bretagne Université de Bretagne Sud LIMATB -UBS -Lorient Centre de Recherche Rue de \\ Saint Maudé - BP 9211656321 Lorient cedex, France \\ ${ }^{3}$ IRSTEA, ETNA - Geomechanics Group, 2, rue de la papeterie 38042 St Martin d'Heres cedex, France \\ ${ }^{4}$ Grenoble Alpes University, 3SR, BP 5338041 Grenoble cedex 9, France
}

Key words Kinematic Structural Stability, Hilbert spaces, kinematic constraint, non self-adjoint operators, Hilbert basis, variational formulation.

In this paper we deal with recent results on divergence kinematic structural stability (ki.s.s.) resulting from discrete nonconservative finite systems. We apply them to continuous nonconservative systems which are shown in the well-known Beck column. When the column is constrained by an appropriate additional kinematic constraint, a certain value of the follower force may destabilize the system by divergence. We calculate its minimal value, as well as the optimal constraint. The analysis is carried out in the general framework of inPnite dimensional Hilbert spaces and non-self-adjoint operators.

\section{Introduction}

Many research papers have recently described the instability issue of non conservative elastic systems with a new point of view (see for example [7,21-29]). They mainly build a bridge between the so-called second order work criterion and the usual Lyapounov stability criterion. The so-called second order work criterion was first introduced by Hill [14] for solid mechanics and intensively used in the framework of non-associated plasticity. Absi [1] also dealt with the link between the two stability criteria in the framework of elastic structural mechanics. However, they did not achieve this junction.

In fact, this junction may be done by observing that one characteristic property of such nonconservative systems is the paradoxical potential destabilizing effect when appropriate kinematic constraints are added. Thompson [42] seems to be the first one to point out this phenomenon. Thompson was concerned by the problem of the stability of fluid conveying tubes whose Beck column is an appropriate model in order to investigate the loss of stability. Because of the wide applications of fluid conveying pipes, it has been and it remains today deeply investigated $([9,10,35,36,38-41,43]$ for example). These papers focus on strong issues like dynamic nonlinearities and especially dynamical bifurcations, complex boundaries conditions, fluide-structure interaction dissipation through visco-elasticity of the fluid or of the structure etc. However, to the best of our knowledge, neither Thompson nor any other previously mentioned researcher did not carry out any investigation about the possible paradoxical destabilizing effect by appropriate additional kinematic constraints.

This paradoxical fact bears similarities with the well-known possible destabilizing effect by additional viscous damping. It has been deeply investigated and references about this paradox are numerous (see only as example $[4,17,18,45])$. These two paradoxical phenomena are both characteristic properties of nonconservativeness of the system. The bridge between the second-order work criterion and the Lyapounov stability is then solved thanks to the kinematic structural stability (also called the ki.s.s.) of an equilibrium. This concept has been recently introduced (see [27-29]) in order to classify the stability issues in relation with the former mentioned paradoxical effect. It has also been investigated in case of divergence and flutter-type instabilities. As far as we are concerned, the major result is that the divergence ki.s.s. is guaranted as long as the second order work holds. Moreover, this criterion is optimal: as soon as the second order work criterion fails, the divergence ki.s.s fails too. There is then a kinematic constraint that destabilizes by divergence the system. There is an effective process to build this constraint. Finally, the number of contraints is inconsequential regarding this divergence ki.s.s. issue. 
The general framework of these developments remains the finite dimension linear algebra. In other words, it deals with discrete mechanical systems in a linear framework. Nonlinear extension has been just recently implemented (see [28]) but for a dual problem. It is then natural to question the possible extension of this set of results to infinite dimension namely to continuum mechanics. It is the central subject of the present paper. Because the 2 d.o.f. Ziegler system is the paradigmatic example that allows us to illustrate the general results obtained in discrete mechanics, we have chosen to tackle this extension with the direct generalization of the Ziegler system (Fig. 1) to continuum mechanics namely with the so-called Beck column (Fig. 2). For pedagogical purposes, we will systematically expose the corresponding results obtained for discrete systems. That will point out what a direct extension is and what elements are specific to the infinite dimension.

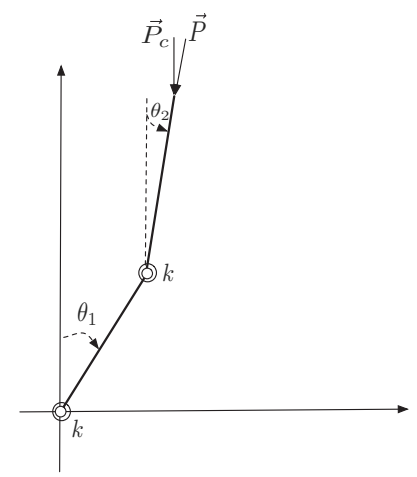

Fig. 12 d.o.f. Ziegler system.

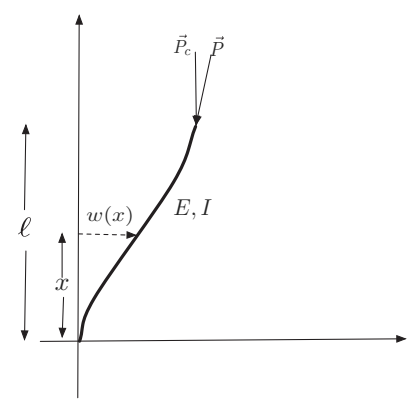

Fig. 2 Beck column.

It is well-known that the Beck column, as well as the Ziegler system, are divergence stable for any value of the complete follower force $P$ acting at their free extremity. Thus, these nonconservative systems are only flutter unstable. The main goal of this paper is to address the following issue: is there a critical value $P^{*}$ of the follower load of a Beck column so that

(1) no additional kinematic constraint may destabilize by divergence the system for $P<P^{*}$,

(2) there is almost one additional kinematic constraint that destabilizes by divergence the system if $P=P^{*}$.

In finite dimension, two equivalent ways may be used to answer the above question. The first one uses an algebraic trick on the determinant of an appropriate matrix. This method cannot then directly be extended to infinite dimension. The second one uses a geometric approach involving other mathematical tools like adjoint mappings, orthogonal projections and compressions of linear maps. These tools may more likely be extended to infinite dimension provided the topological issues are taken into account. We have chosen this approach.

However, the tools used in a finite dimension have two forms, namely linear and bilinear. They are equivalent in a finite dimension framework. In infinite dimension, they are not the same and lead to a strong formulation for the former and to a weak formulation for the latter. As usual, the weak formulation is the most appropriate one and, as far as possible, we bypass the operator language and we use it only to concretely make the computations when required.

Some of these algebraic tools have already been used in the same framework of nonconservative continuous systems by researchers about fifty years ago. For example, it is worth noting that (see for example $[13,34,37]$ ) the adjoint of the differential operator arising in stability problems of continuum mechanics has then been intensively used but essentially in order to describe the flutter unstable modes better than with the help of the Ritz-Galerkin approximation method. Unfortunately, because they have not been aware of the kinematic structural stability issue, these authors have not realized that a similar way could also be followed to compute the critical load of the kinematic destabilization by divergence. In this paper, similar calculations to get the critical load and its corresponding critical kinematic constraint as well are performed. The above mentioned authors used the adjoint of the initial operator whereas we use its symmetric and skew symmetric parts.

Because of the infinite dimension of the involved spaces, some new topological issues naturally arise. They are solved by the use of the language of Hilbert spaces which is the most appropriate for this set of questions. We will recall some useful and general results and notations when appropriate so as to specify the differences with the finite dimension framework. The reader, familiar with these aspects can bypass these developments. They are provided only for highlighting the extension from a finite to an infinite dimension. For developments and synthetic views of the topics involved in this paper, see for example $[20,30]$. 
The paper is organized as follows. Firstly, the issue about the Beck column is formulated and the results about the corresponding discrete system, namely the 2 d.o.f. Ziegler system are recalled. Secondly, some elements of the language of Hilbert spaces used later for tackling the issue are pointed out. A special attention is paid to kinematic constraints in this framework. Thirdly, we highlight the systematic machinery that may be used to tackle the divergence ki.s.s. for any continuous system. As an application, we solve the issue for the Beck column. Questions linked with infinite dimension (choice of norms, compactness of operators, regularity of solutions,... ) are accurately investigated. A last section deals with the link with stability analysis. A substantial literature exists related to Lyapounov method of stability analysis. In the infinite dimensional framework, Mochvan and his followers (see [19,31,32] or [33] for example) showed the dependency of this method on the choice of the used metric, necessitating generally a special attention. However, it should be stressed that our investigations are only linear. In this linear framework, we first bring to light a unbounded solution of the dynamic equation of the constrained system for the critical value $P=P^{*}$. Thanks to the conservative nature of the constrained system, the stability of the constrained system is then ensured for $P<P^{*}$.

\section{What is the issue?}

\subsection{Strong formulation of divergence stability of the Beck column and its Ziegler system counterpart}

The goal of this paper is then to generalize the results obtained recently for discrete elastic systems to continuous systems. Thus, we investigate the possibility to destabilize by divergence the Beck column thanks to appropriate additional kinematic constraints. For a better understanding of the mathematical process used, the results for the analogous $n=2$ d.o.f. system, namely for the Ziegler system, are mentioned in parallel. Note, however, that these results are valid for any finite discrete system or in other words in a finite dimension, even if we choose $n=2$ to simplify our demonstration.

The basic equations of the Beck column with their hypotheses are supposed well-known: linear elasticity, small displacements and small strains (Lagrange and Euler variables identified), Euler-Bernouilli kinematic model, vertical equilibrium configuration. $P$ is the follower (nonconservative) load at the free extremity $(x=\ell)$ ). The unknown is the transversal displacement $x \mapsto w(x)$ with respect to the equilibrium configuration (see Fig. 2). The equilibrium equation reads:

$$
\left.A(w)=A(P)(w)=E I w^{\prime \prime \prime \prime}(x)+P w^{\prime \prime}(x)=0 \quad \forall x \in\right] 0, \ell[
$$

and the boundary conditions read:

$$
w(0)=0, \quad w^{\prime}(0)=0, \quad E I w^{\prime \prime}(\ell)=0, \quad E I w^{\prime \prime \prime}(\ell)=0 .
$$

In the finite dimension case $(n=2$, see Fig. 1), the finite dimensional equivalent issue of $(1)+(2) \operatorname{reads} K(P) X=0$ where $X=\left(\begin{array}{l}\theta_{1} \\ \theta_{2}\end{array}\right)$ is the analogous of $w$ and

$$
K(P)=\left(\begin{array}{cc}
2 k-P \frac{\ell}{2} & -k+P \frac{\ell}{2} \\
-k & k
\end{array}\right) \in \mathcal{M}_{2}(\mathbb{R})
$$

which is the analogous of the differential operator $A=A(P)$ appearing in (1). Obviously, there is no boundary condition for discrete systems.

An usual (but instructive) calculation shows that, for all $P$, the unique solution of $(1)+(2)$ is $w=0$ which means that no divergence instability may occur and that the Beck column is only flutter unstable.

Similarly, in the finite dimensional case $(n=2$ see Fig. 1$)$, $\operatorname{det}(K(P))=k^{2}$ is independent of $P$ which shows that $K(P)$ is invertible for all $P$. Thus, $K(P) X=0$ implies $X=0$ also for all $P$ which means that no divergence instability may occur and that the Ziegler column is also only flutter unstable.

The difference with a vertical conservative dead load $P_{c}$ at $x=\ell$ (see Fig. 2) lies in the last boundary condition. For a follower nonconservative force, the boundary condition reads $E I w^{\prime \prime \prime}(\ell)=0$ whereas, for the conservative load, this condition reads $E I w^{\prime \prime \prime}(\ell)+P_{c} w^{\prime}(\ell)=0$. For a conservative load $P_{c}$, the whole following system of boundary conditions then read:

$$
w(0)=0, \quad w^{\prime}(0)=0, \quad E I w^{\prime \prime}(\ell)=0, \quad E I w^{\prime \prime \prime}(\ell)+P_{c} w^{\prime}(\ell)=0 .
$$

This differential boundary problem admits a not nil solution $w$ for the lowest continuous conservative buckling critical load

$$
P_{c}=\pi^{2} \frac{E I}{4 \ell^{2}}
$$


In the finite dimensional case ( $n=2$, see Fig. 1) and for a conservative dead load $P_{c}$, the stiffness matrix then reads $K_{c}\left(P_{c}\right)=\left(\begin{array}{cc}2 k-P_{c} \frac{\ell}{2} & -k \\ -k & k-P_{c} \frac{\ell}{2}\end{array}\right) \in \mathcal{M}_{2}(\mathbb{R})$ which leads to the discrete conservative buckling critical load

$$
P_{c}^{*}=(3-\sqrt{5}) \frac{k}{\ell}
$$

\subsection{Usual weak formulation of divergence stability of the Beck column and its Ziegler system counterpart}

Let $\mathcal{U}$ be the vector space of functions $\in \mathcal{C}^{4}([0, \ell])$ satisfying the boundary conditions (2). Let $w \in \mathcal{U}$ a solution of (1) + (2). After multiplying by a test function $v$ (for example $\in \mathcal{C}^{\infty}([0, \ell])$ with compact support) and after two integrations by parts, the variational formulation of the same problem reads:

Find $w \in \mathcal{V}$ such that

$$
\phi(w, v)=\phi(P)(w, v)=\int_{0}^{\ell}\left(E I w^{\prime \prime}(x) v^{\prime \prime}(x)+P w^{\prime \prime}(x) v(x)\right) d x=0 \quad \forall v \in \mathcal{V}
$$

where $\mathcal{V}$ is the Hilbert space of functions $v \in \mathcal{L}^{2}([0, \ell])$ whose first and second derivatives are also in $\mathcal{L}^{2}([0, \ell])$ and which satisfy the kinematic boundary conditions $v(0)=v^{\prime}(0)=0$. There is a real difficulty with the value of a function of $L^{2}([0, \ell])$ at a point because they are classes of functions and not really continuous functions. In the case of functions of one single variable, one proves that an element of $\mathcal{V}$ is continuous (has a continuous represent) with a continuous derivative. Thus, we do not need the more subtile theory of the trace on the boundary. By integrating another time by parts the second term of (5), the variational formulation of the problem also reads:

Find $w \in \mathcal{V}$ such that

$$
\phi(w, v)=\phi(P)(w, v)=\int_{0}^{\ell}\left(E I w^{\prime \prime}(x) v^{\prime \prime}(x)-P w^{\prime}(x) v^{\prime}(x)\right) d x+P w^{\prime}(\ell) v(\ell) \quad \forall v \in \mathcal{V} .
$$

The nonconservativeness of the problem is clearly reflected in this variational formulation by the loss of symmetry of the bilinear form $\phi(P)$ on $\mathcal{V}$ in (5) (or (6) as well). For the conservative load, we get the following symmetric bilinear form $\phi_{c}=\phi_{c}(P)$ on the same space $\mathcal{V}$ because of the same kinematic conditions at $x=0$ :

$$
\phi_{c}(w, v)=\phi_{c}(w, v)(P)(w, v)=\int_{0}^{\ell}\left(E I w^{\prime \prime}(x) v^{\prime \prime}(x)-P w^{\prime}(x) v^{\prime}(x)\right) d x \quad \forall w, v \in \mathcal{V} .
$$

We will use the usual vocabulary of Structural Mechanics: kinematic boundary conditions are called essential boundary conditions (here $w\left(0=w^{\prime}(0)=0\right.$ ) whereas the dynamic boundary conditions are called natural boundary conditions (here $\left.E I w^{\prime \prime}(\ell)=0, E I w^{\prime \prime \prime}(\ell)=0\right)$.

In the finite dimensional case $(n=2)$, the non symmetry of $K(P)=\left(\begin{array}{c}2 k-p \frac{\ell}{2}-k+P \frac{\ell}{2} \\ -k\end{array}\right) \in \mathcal{M}_{2}(\mathbb{R})$ shows the nonconservativeness of the Ziegler system whereas, for the conservative load $P_{c}$, the stiffness matrix $K_{c}\left(P_{c}\right)=\left(\begin{array}{c}2 k-P_{c} \frac{\ell}{2} \\ -k \\ k-P_{c} \frac{\ell}{2}\end{array}\right) \in \mathcal{M}_{2}(\mathbb{R})$ is symmetric.

The equation $K(P) X=0$ is equivalent to $Y^{T} K(P) X=0$ for all $Y=\left(\begin{array}{l}y_{1} \\ y_{2}\end{array}\right) \in \mathcal{M}_{21}(\mathbb{R})$ which is the 2 dimensional counterpart of (5).

Let us sum up the similarities and the differences between the finite $(n=2$ here) and infinite dimensional cases.

1. The same operator $K(P)$ on $\mathcal{M}_{21}(\mathbb{R})$ in a finite dimension has two counterparts in an infinite dimension:

(a) The differential operator $A(P)$ on the space $\mathcal{U}$ when $K(P)$ is viewed as the linear map $X \mapsto K(P) X$ on $\mathcal{M}_{21}(\mathbb{R})$.

(b) The bilinear form $\phi(P)$ on $\mathcal{V}$ when $K(P)$ is viewed as the bilinear map $(X, Y) \mapsto Y^{T} K(P) X$ on $\mathcal{M}_{21}(\mathbb{R})$.

2. The nonconservativeness viewed as the loss of symmetry of the matrix $K(P)$ (identical for the both viewpoints in a finite dimension) is however better highlighted by the variational formulation with $\phi(P)$ on $\mathcal{V}$.

3. In a finite dimension, the divergence stability of the system for any value of $P$ means that $K(P)$ is invertible in $\mathcal{M}_{2}(\mathbb{R})$ for all $P$ or equivalently that it is an injective map on $\mathcal{M}_{21}(\mathbb{R})$. In an infinite dimension, it only means that $A(P)$ is an injective map on $\mathcal{U}$. Its counterpart is then formulated thanks to a linear operator.

4. The infinite dimension leads to different vector spaces for both the formulations: $\mathcal{U}$ for the linear (strong) formulation and $\mathcal{V}$ for the bilinear (weak) formulation. These formulations are not equivalent but any $w \in \mathcal{U}$ (strong) solution of $A(P)(w)=0$ is also a (weak) solution of the weak formulation. $A(P)$ is injective for all $P$. Thus, $w=0$ is the only solution of the strong formulation. 
An essential step in Functional Analysis is to show that a weak solution is also a strong solution. In fact, the weak and variational formulation is an expression of the equilibrium equation according to the Virtual Power Principle. Thus, one has to prove that:

Issue 1: Is $w=0$ the only solution of the weak formulation, namely:

$$
\phi(P)(w, v)=0 \quad \forall v \in \mathcal{V} \Longrightarrow w=0 \quad(\in \mathcal{V}) ?
$$

$w=0$ necessarily means that $w(x)=0 \forall x \in[0, \ell]$ because of the property of functions of $\mathcal{V}$ mentioned above: the continuous representing of $w$ vanishes identically, namely $w$ is the nil function. This issue will be solved in (2.3).

\subsection{Results in a finite dimension and position of the problem}

We now summarize the recent results obtained in the finite dimensional framework. They are presented within the 2 dimensional framework.

1. For the minimal positive value $\tilde{P}^{*}=4 \frac{k}{\ell}$, there is a destabilizing kinematic constraint. $\tilde{P}^{*}$ is the minimal positive root of the equation

$$
\operatorname{det} K_{s}(P)=k^{2}-\frac{P^{2} \ell^{2}}{16}=0
$$

where $K_{S}=K_{s}(P)=\frac{1}{2}\left(K(P)+K^{T}(P)\right)=\left(\begin{array}{cc}2 k-P \frac{\ell}{2} & -k+P \frac{\ell}{4} \\ -k+P \frac{\ell}{4} & k\end{array}\right)$ is the symmetric part of $K(P)$. The destabilizing constraint $\tilde{C}^{*}$ viewed as a vector of $\mathcal{M}_{21}(\mathbb{R})$ must be chosen in $K\left(\tilde{P}^{*}\right)^{-1}\left(\operatorname{ker} K_{s}\left(\tilde{P}^{*}\right)\right)$ which exists because $K(P)$ is invertible for all $P$.

2. By construction, $\tilde{C}^{*}$ is in $K_{a}\left(\tilde{P}^{*}\right)^{-1}\left(\operatorname{ker} K_{s}\left(\tilde{P}^{*}\right)\right)$. For the 2 d.o.f. Ziegler system and for the critical value $\tilde{P}^{*}=$ $4 \frac{k}{\ell}$, one finds $K_{s}\left(\tilde{P}^{*}\right)=\left(\begin{array}{ll}0 & 0 \\ 0 & k\end{array}\right)$. Then $\operatorname{ker} K_{s}\left(\tilde{P}^{*}\right)$ is the one dimensional subspace generated by $\tilde{X}^{*}=\left(\begin{array}{l}1 \\ 0\end{array}\right)$. For the critical value $\tilde{P}^{*}=4 \frac{k}{\ell}$, $\operatorname{ker} K_{s}\left(\tilde{P}^{*}\right)$ is the one dimensional subspace generated by $\tilde{X}^{*}=\left(\begin{array}{l}1 \\ 0\end{array}\right)$. Then, $K_{a}\left(\tilde{P}^{*}\right)\left(\tilde{X}^{*}\right)=$ $\left(\begin{array}{cc}0 & -\tilde{P}^{*} \frac{\ell}{4} \\ -P^{*} \frac{\ell}{4} & 0\end{array}\right)\left(\begin{array}{l}1 \\ 0\end{array}\right)=\left(\begin{array}{c}0 \\ -k\end{array}\right)$. The destabilizing constraint then reads $-k \theta_{2}=0$ or more simply $\theta_{2}=0$.

Remark that any kinematic constraint reads $a_{1} \theta_{1}+a_{2} \theta_{2}=0$ and may be identified with the vector $C=\left(\begin{array}{l}a_{1} \\ a_{2}\end{array}\right)$ so that the constraint reads $C^{T} X=0$. Any constraint is then a linear form $c: Y \mapsto c(Y)$ on $\mathcal{M}_{21}(\mathbb{R})$ which has the form $Y \mapsto C^{T} Y$ with a vector $C \in \mathcal{M}_{21}(\mathbb{R})$. This is the Riesz's representation theorem.

3. The last result is the optimal property of this above algebraic procedure. As long as $P<\tilde{P}^{*}$, no additional kinematic constraint may destabilize the system. The first proof of this results has been established from a trick (see [7,21]) using the determinant of the augmented system

$$
\left\{\begin{aligned}
K(P) X+\lambda C & =0 \\
C^{T} X & =0
\end{aligned}\right.
$$

with $\lambda$ the Lagrange multiplier associated to the kinematic constraint defined by $Y \rightarrow C^{T} Y$. We now recall the main steps of the proof to highlight the meaning of the result and the used tools. The following block calculation

$$
\left(\begin{array}{cc}
K(P) & C \\
C^{T} & 0
\end{array}\right)\left(\begin{array}{cc}
K^{-1}(P) & -K^{-1}(P) A \\
0 & 1
\end{array}\right)=\left(\begin{array}{cc}
I_{2} & 0 \\
C^{T} K^{-1}(P) & -C^{T} K^{-1}(P) C
\end{array}\right)
$$

shows that the constrained system described by (8) is divergence unstable by an additional kinematic constraint defined by $Y \mapsto C^{T} Y$ if and only if $C^{T} K^{-1}(P) C=0$. Thanks to the fact that $Z \mapsto K^{-1}(P) Z$ is an isomorphism of $\mathcal{M}_{21}(\mathbb{R}), C^{T} K^{-1}(P) C=0$ is successively equivalent to $C^{T} K^{-1}(P)^{T} K(P)^{T} K^{-1}(P) C=0$ or $\left(K^{-1}(P) C\right)^{T} K(P)^{T}\left(K^{-1}(P) C\right)=0$ or again

$$
\left(K^{-1}(P) C\right) K(P)\left(K^{-1}(P) C\right)=0,
$$

namely $D^{T} K(P) D=0$ with $D=K^{-1}(P) C$ or $C=K(P) D$ which is equivalent to

$$
D^{T} K_{S}(P) D=0 .
$$

This last equation reflects the failure of the second-order work criterion for $K(P)$ which means that $K(P)$ or $K_{s}(P)$ are no longer definite. But, for $P=0$, the system is conservative stable which implies that the matrix $K(0)=K_{s}(0)$ is a symmetric definite positive matrix. Its eigenvalues are then real strictly positive. Thus the eigenvalues of $K_{S}(P)$ must pass through 0 in order that the signature of the quadratic form associated with $K_{s}(P)$ changes (continuity 
argument): the value $\tilde{P}^{*}$ that makes degenerate $K_{s}(P)$ as a quadratic form is also the value making $K_{s}(P)$ singular as a matrix. $\tilde{P}^{*}$ is then the lowest positive $\operatorname{root}$ of $\operatorname{det} K_{S}(P)=0$.

Two questions may be worth addressing within the infinite dimensional framework:

Issue 2: Is there an optimal kinematic constraint that may destabilize the Beck column and if so, for which minimal positive value $P^{*}$ of $P$ ?

Issue 3: For the value $P^{*}$, find a corresponding optimal destabilizing kinematic constraint.

However, the above methods, performing in a finite dimension cannot be directly generalized to the infinite dimension. It is due to the involved algebraic tools like the determinant. Another way must be followed. However, before tackling the issues 1, 2, and 3, we now stress some usual topological aspects of the Hilbert approach of Structural Mechanics.

\section{Fundamental Spaces - Topological aspects. Variational formulation of the problem}

\subsection{Vector spaces and Operator $A(P)$. Usual aspects}

Let $j: \mathcal{V} \rightarrow \mathcal{L}^{2}([0, \ell])$ be the canonical injection. To highlight topological properties of $j$, we introduce different norms and topologies on these spaces. We will use the natural Hilbert structures on $\mathcal{V}$ and on $\mathcal{L}^{2}([0, \ell])$.

$\mathcal{V}$ is equipped with the scalar product $\left(\|.\|_{\mathcal{V}}\right.$ is the associated norm)

$$
(w \mid v)_{\mathcal{V}}=\int_{0}^{\ell}\left(w^{\prime \prime}(x) v^{\prime \prime}(x)+w^{\prime}(x) v^{\prime}(x)+w(x) v(x)\right) d x
$$

and $\mathcal{L}^{2}([0, \ell])$ is equipped with the natural scalar product $(\|$.$\| is the associated norm)$

$$
(w \mid v)=\int_{0}^{\ell} w(x) v(x) d x .
$$

The following topological properties hold:

1. $j$ is a continuous compact map because the induced topology of $\|$. $\|$ on $\mathcal{V}$ by the one of $\mathcal{L}^{2}([0, \ell])$ is weaker than the natural one of $\mathcal{V}$ namely $\|.\|_{\mathcal{V}}$. The continuity of $j$ means that there is a constant $k$ such that $\|j(w)\| \leq$ $k\|w\|_{\mathcal{V}} \forall w \in \mathcal{V}$. The compactness of $j$ means that $j(B)\left(B=B_{\mathcal{V}}\right.$ is the unit ball of $\left.\mathcal{V}\right)$ is a relatively compact set of $\mathcal{L}^{2}([0, \ell])$. This property is often used to prove the compactness of operators and to investigate their spectral properties.

2. $\mathcal{V}$ is a dense subvectorspace of $\mathcal{L}^{2}([0, \ell])$ obviously for $\|$.$\| .$

3. $\phi$ is a continuous bilinear map on $\mathcal{V}$ which means that there is a constant $c$ such that $|\phi(P)(w, v)| \leq$ $c\|w\|_{\mathcal{V}}\|v\|_{\mathcal{V}} \quad \forall w, v \in \mathcal{V}$.

In the above first section, we started with the usual differential equation of the equilibrium of a Beck column. It is an operator $A(P)$ with its boundary conditions and its space of $\mathcal{U}$ of strong solutions. We then deduced the weak formulation. Now, thanks to the previous topological considerations, one can recapture the operator $A(P)$ and $\mathcal{U}$ from the weak or variational formulation defined by the bilinear map $\phi(P)$ on $\mathcal{V}$.

Indeed, let $\mathcal{U}_{\phi}$ the subspace of $\mathcal{V}$ built by the elements $w \in \mathcal{V}$ such that $v \mapsto \phi(P)(w, v)$ is continuous on $\mathcal{V}$ but for the induced topology by $\mathcal{L}^{2}([0, \ell])$ namely by $\|$.$\| . That is not in contradiction with the last point 3$ because the continuity for $\|.\| \mathcal{V}$ does not implies the continuity for $\|$.$\| .$

By the Riesz representation theorem, if $w \in \mathcal{U}_{\phi}$ there is a unique vector $A_{\phi}(P)(w) \in \mathcal{L}^{2}([0, \ell])$ such that

$$
\phi(P)(w, v)=\left(A_{\phi}(P)(w) \mid j(v)\right) \forall v \in \mathcal{V}
$$

and a double integration by parts in the opposite sense as above shows, by unicity of the vector $A(P)(w)$, that $\mathcal{U}_{\phi}=\mathcal{U}$ and $A_{\phi}(P)(w)=A(P)(w)$.

For a Beck column, the subspace $\mathcal{U}$ is then:

$$
\mathcal{U}=\left\{v \in \mathcal{V} \mid v \in \mathcal{C}^{4}([0, \ell]), \quad E I w^{\prime \prime}(\ell)=0, \quad E I w^{\prime \prime \prime}(\ell)=0\right\} .
$$

The above considerations are the usual ones in the Hilbert approach of structural mechanics. Note however that, in the usual framework, $\phi(P)$ should be a symmetric definite positive bilinear form and even often a coercive form on $\mathcal{V}$. Coercivity of 
$\phi(P)$ means that there is $\alpha>0$ such that $\phi(v, v) \geq \alpha\|v\|_{\mathcal{V}}^{2}$. This strong property ensures the definite positiveness of $\phi(P)$ defined by: $\phi(P)(w, w)=0 \Longrightarrow w=0$. This last property is nothing else that the second order work criterion.

On may prove that $\mathcal{U}$ is a dense subspace of $\mathcal{V}$ and of $\mathcal{L}^{2}([0, \ell])$. Moreover, $A(P): \mathcal{U} \rightarrow \mathcal{L}^{2}([0, \ell])$ is a linear map but non necessarily bounded.

Regarding mechanical issues, the usual methodology in functional analysis involves starting with the variational (namely weak) formulation, then finding a solution in this framework and finally proving that this solution is "strongly regular" and is a solution of the strong issue. The involved problem necessitates now to focus on the topic of kinematic constraints.

\subsection{Kinematic constraints}

Because of the well adapted weak or variational formulation, kinematic constraints are formulated in this language. Thus, a kinematic constraint is represented by a continuous linear form on $\mathcal{V}$ namely any element $c$ of the topological dual space $\mathcal{V}^{\prime}$. The continuity of linear forms means the continuity for the topology of $\mathcal{V}$ namely for $\|.\| \mathcal{V}$. By the Riesz theorem on the Hilbert space $\mathcal{V}$, any constraint $c: \mathcal{V} \rightarrow \mathbb{R}$ is then represented by a function $f_{c}:[0, \ell] \rightarrow \mathbb{R} \in \mathcal{V}$ such that:

$$
c(v)=\left(f_{c} \mid v\right)_{\mathcal{v}}=\int_{0}^{\ell}\left(f_{c}^{\prime \prime}(x) v^{\prime \prime}(x)+f_{c}^{\prime}(x) v^{\prime}(x)+f_{c}(x) v(x)\right) d x .
$$

This general form of the kinematic constraints is not very intuitive and we now make some additional comments to this central point. In fact, if $a_{1}, a_{2}, a_{3}$ are three elements of $\mathcal{L}^{2}([0, \ell])$ then the linear form

$$
c(v)=\int_{0}^{\ell}\left(a_{1}(x) v^{\prime \prime}(x)+a_{2}(x) v^{\prime}(x)+a_{3}(x) v(x)\right) d x
$$

is continuous on $\mathcal{V}$. This results from the Cauchy-Schwarz inequality and from the fact that the topology on $\mathcal{V}$ is stronger than the one on $\mathcal{L}^{2}([0, \ell])$. If $v \in \mathcal{V}$, then $|c(v)| \leq\left(|| a_{1}\|+\| a_{2}\|+\| a_{3}||\right)\|v\| \mathcal{V}$ which proves the result.

In fact, the Poincaré inequality shows that the norms $v \rightarrow\|v\|_{\mathcal{V}}$ and $v \rightarrow\left\|v^{\prime \prime}\right\|=\|v\|_{\mathcal{V}, 2}$ are topologically equivalent on $\mathcal{V}$. This means that the linear continuous forms on $\mathcal{V}$ are the same for the both norms. So, by applying the Riesz theorem on the Hilbert space $\mathcal{V}$ with this new norm $\|.\|_{\mathcal{V}, 2}$ (and (.|.) $\mathcal{V}, 2_{\text {, }}$ the associated scalar product.), any element $c \in \mathcal{V}^{\prime}$ (namely any additional kinematic constraint) may be also represented by the third following integral form:

$$
c(v)=\int_{0}^{\ell} a^{\prime \prime}(x) v^{\prime \prime}(x) d x=\left(a_{c} \mid v\right)_{\mathcal{V}, 2}
$$

with $a$ element of $\mathcal{V}$. Regarding the investigated issue, the optimal constraint must take this form. The equivalence of these three forms is not obvious at first sight but it is consequences to Poincaré inequality and Riesz representation theorem. Note first that Poincare inequality is often given for functions in $H_{0}^{1}(I)$ with $I$ bounded. In fact, according to the proof of this inequality, it is sufficient that the function vanishes only at one extremity. It is what happens in our problem for both $v$ and $v^{\prime}$ at $x=0$. In the appendix, we show explicitly the equivalence of the last two forms (14) and (15) of any constraint $c \in \mathcal{V}^{\prime}$.

\subsection{An alternative formulation involving only the Hilbert space $\mathcal{V}$ equipped with $(. \mid \cdot) \mathcal{V}, 2$. Solution of issue 1}

We now reformulate the problem by involving only the Hilbert space $\mathcal{V}$ equipped with $(. \mid.) \mathcal{V}, 2$. Thanks to the continuity of the bilinear form $\phi(P)$ on $\mathcal{V}$, there is, for each $w \in \mathcal{V}$, a unique vector denoted $\tilde{A}(P)(w)$ such that $\phi(P)(w, v)=$ $(\tilde{A}(P)(w) \mid v)_{\mathcal{V}, 2}$ for all $v \in \mathcal{V}$. Moreover, $\tilde{A}(P)$ is a continuous linear map of $\mathcal{V}: \tilde{A}(P) \in \mathcal{L}_{c}(\mathcal{V})$. This formulation does not necessitate to introduce simultaneously a domain as for the operator $A(P)$ defined thanks to the more usual scalar product of $\mathcal{L}^{2}[0, \ell]$. Both calculations will be performed for the central bilinear form of our paper which is not $\phi(P)$ but its symmetric part $\phi_{s}(P)$. That illustrate the two aspects and their mutual relationship.

However, we now take advantage of this new formulation, by solving the issue 1 formulated above. Indeed, $\phi(P)(w, v)=$ $0 \forall v \in \mathcal{V} \Longrightarrow w=0 \quad(\in \mathcal{V})$ is equivalent to $\tilde{A}(P)(w)=0 \Longrightarrow w=0(\in \mathcal{V})$ namely that $\tilde{A}(P)($ and not $A(P))$ is an injective element of $\mathcal{L}_{c}(\mathcal{V})$. We now calculate the operator $\tilde{A}(P)$.

\subsubsection{Calculation of $\tilde{\boldsymbol{A}}(\boldsymbol{P})$}

By definition, if $w \in \mathcal{V}, \tilde{A}(P)(w)$ is the unique function $\in \mathcal{V}$ such that

$$
\phi(P)(w, v)=(\tilde{A}(P)(w) \mid v)_{\mathcal{V}, 2}=\int_{0}^{\ell}(\tilde{A}(P)(w))^{\prime \prime}(x) v^{\prime \prime}(x) d x .
$$


Calculations read successively after integrations by parts and using that $w(0)=w^{\prime}(0)=v(0)=v^{\prime}(0)=0$ since $w$ and $v$ are in $\mathcal{V}$ :

$$
\begin{aligned}
\phi(P)(w, v) & =\int_{0}^{\ell}\left(E I w^{\prime \prime}(x) v^{\prime \prime}(x)+P w^{\prime \prime}(x) v(x)\right) d x \\
& =\int_{0}^{\ell}\left(E I w^{\prime \prime}(x) v^{\prime \prime}(x)+P w(x) v^{\prime \prime}(x)\right) d x+P w^{\prime}(\ell) v(\ell)-P w(\ell) v^{\prime}(\ell) \\
& =\int_{0}^{\ell}(\tilde{A}(P)(w))^{\prime \prime}(x) v^{\prime \prime}(x) d x
\end{aligned}
$$

with

$$
\tilde{A}(P)(w)(x)=E I w(x)+P \int_{0}^{x}\left(\int_{0}^{t}\left(w(s)+(\ell-s) w^{\prime}(\ell)-w(\ell)\right) d s\right) d t .
$$

One may easily check that $\tilde{A}(P)(w) \in \mathcal{V}$ and that $\tilde{A}(P) \in \mathcal{L}_{c}(\mathcal{V})$.

\subsubsection{Solution of issue 1}

The following proposition addresses the issue 1 .

Proposition 1. For all $P \in \mathbb{R}, \tilde{A}(P)$ is injective element of $\mathcal{L}_{c}(\mathcal{V})$.

Pro o f. Let $w \in \operatorname{ker} \tilde{A}(P)$. Thus, $w \in \mathcal{V}, \tilde{A}(P)(w)=0 \in \mathcal{V}$. We deduce that

$$
E I w(x)=-P \int_{0}^{x}\left(\int_{0}^{t}\left(w(s)+(\ell-s) w^{\prime}(\ell)-w(\ell)\right) d s\right) d t
$$

and this relation holds not only almost everywhere but also for all $x \in[0, \ell]$ because of the continuity of $w$. The right-hand side of (17) is twice continuously differentiable with respect to $x \in] 0, \ell\left[\right.$ and thus, $w$ too. But, since $\left.w \in \mathcal{C}^{2}[0, \ell]\right)$, the right-hand side of (17) is four times continuously differentiable and so on. We deduce that $\left.w \in \mathcal{C}^{\infty}[0, \ell]\right)$. Making the derivative of (17) twice leads to $E I w^{\prime \prime}(x)=-P\left(w(x)+(\ell-x) w^{\prime}(\ell)-w(\ell)\right)$ for all $x \in[0, \ell]$. We first deduce that $E I w^{\prime \prime}(\ell)=0$. Successively one and two further derivatives lead first to $E I w^{\prime \prime \prime}(\ell)=0$ and then to $E I w^{\prime \prime \prime \prime}(x)=-P w^{\prime \prime}(x)$ $x \in[0, \ell]$. Then $w$ is a solution of (1) and (2) and thus, $w=0$.

\subsection{Variational formulation for the ki.s.s problem}

In a finite dimension, he solution has been first reached thanks to the strong formulation and the use of the Lagrangian multiplier (8) but this way is not appropriate to the infinite dimensional framework. On the contrary, the method will now involve a weak formulation of the problem without Lagrange multiplier. Nevertheless, both formulations are equivalent in a finite dimension ([26]) and, in addition, the trick using the determinant of block matrices finally leads to remove the Lagrange multiplier.

Let $c \in \mathcal{V}^{\prime}$ be a continuous linear form representing an additional kinematic constraint. $F_{c}=\operatorname{ker} c$ is a closed subspace of $\mathcal{V}$ and we denote $p_{F_{c}}$ the orthogonal projection on $F_{c}$ thanks to the decomposition $\mathcal{V}=F \perp F^{\perp}$ for any closed $F$ of a Hilbert space. From now on, the orthogonality involves the scalar product (.|. $)_{\mathcal{V}, 2}$ on $\mathcal{V}$. We then are looking for $c \in \mathcal{V}^{\prime}$ such that

$$
\phi(P)(w, v)=0 \quad \forall v \in F_{c}
$$

admits a not nil solution $w \in F_{c}$. It is equivalent to find $c \in \mathcal{V}^{\prime}$ such that

$$
\phi(P)(w, v)=\lambda c(v) \quad \forall v \in \mathcal{V}
$$

admits a not nil solution $w \in F_{c}$ where $\lambda \in \mathbb{R}$ is the Lagrange multiplier associated with the constraint $c$.

Let $\tilde{A}(P)$ be the operator defined as above and let $a_{c} \in \mathcal{V}$ be the unique element such that $c(v)=\left(a_{c} \mid v\right)_{\mathcal{V}, 2}$ for all $v \in \mathcal{V}$. Thus $F_{c}=a_{c}^{\perp}$ and (18) is equivalent to finding $a_{c}$ in $\mathcal{V}$ such that:

$$
\exists w \neq 0 \perp a_{c} \quad \tilde{A}(P)(w) \in F_{c}^{\perp}
$$

or

$$
\exists w \in F_{c} \backslash\{0\} \quad \tilde{A}(P)(w) \in F_{c}^{\perp} .
$$


(19) is equivalent to find $a_{c} \in \mathcal{V}$ so that

$$
\exists w \neq 0 \perp a_{c} \quad \tilde{A}(P)(w)=\lambda a_{c}
$$

with $\lambda \in \mathbb{R}$

\subsection{Geometric solution of the ki.s.s. issue: compression of an operator}

[26] and [29] showed the equivalence between the first solution of the ki.s.s. issue involving the augmented system with Lagrangian multiplier and block matrix products and a second more geometric solution based upon the concept of compression of an operator. We then provide this last approach.

Let $F$ be any closed subset of a Hilbert space $\mathcal{H}$. The orthogonal projector $p_{F}$ is a self-adjoint continuous operator of $\mathcal{H}$ and the compression $u_{F}$ of $F$ is $p_{F} \circ u_{\mid F} \in \mathcal{L}_{c}(F)$ where $u_{\mid F}$ is the restriction of $u$ to $F$. Regardess of the ki.s.s. issue, the main result about the compressions remains valid in the infinite dimension and reads:

Theorem 2. Any compression (on closed subspaces) of an injective continuous linear map $u \in \mathcal{L}_{c}(\mathcal{H})$ remains injective if and only if $u\left(\right.$ or $\left.u_{s}\right)$ is definite.

(To best of our knowledge, no paper or book mention this result.)

$\operatorname{Pr}$ o o $\mathrm{f}$. First let us suppose that $u$ is definite and that there is a closed subspace $F$ of $\mathcal{H}$ with $u_{F}$ non injective. Thus, there is $x \in F$ with $u_{F}(x)=0$ and then $\left(u_{F}(x) \mid x\right)=0=\left(p_{F} \circ u(x) \mid x\right)=\left(u(x) \mid p_{F}^{*}(x)\right)=\left(u(x) \mid p_{F}(x)\right)=(u(x) \mid x)$ (because $x \in F$ ) which signs that $u$ is non definite: contradiction.

Reciprocally, let us suppose that any compression of $u \in \mathcal{L}(\mathcal{H})$ is injective. Let $x$ be $\neq 0$ and let $F$ be the subspace spanned by $x$. Thus, thanks to the hypothesis, $u_{F}(x)=p_{F} \circ u(x) \neq 0$. Thus, $u_{F}(x)=(u(x) \mid x) x \neq 0$ which implies that $(u(x) \mid x) \neq 0: u$ (namely $\left.u_{s}\right)$ is definite.

We now apply the concept of compression to the ki.s.s. issue. Let $c \in \mathcal{V}^{\prime}$ be an additional kinematic constraint defined as above by the vector $a_{c}$ so that $F_{c}=\operatorname{ker} c=<a_{c}>^{\perp}$. Let $\tilde{A}_{F_{c}}(P)$ be the compression of $\tilde{A}(P)$ on $F_{c}$. Then, (21) means that $\tilde{A}_{F_{c}}(P)(w)=0$ for a vector $w \in F_{c} \backslash\{0\}$ and then $\tilde{A}_{F_{c}}(P)$ is not injective. According to theorem $(2), \tilde{A}_{s}$ is not definite. We then proved:

Proposition 3. The ki.s.s holds as long as any compression on codimension 1 subspaces of Ã is injective or equivalently as long as $\tilde{A}_{s}$ is definite (positive). In other words, the second order work criterion guaranted the divergence ki.s.s.

Suppose now that the operator $u \in \mathcal{L}_{c}(\mathcal{H})$ continuously depends on a parameter $P \geq 0$ so that $u(0)$ is a self-adjoint coercive operator of $\mathcal{H}$. It follows that $u(0)=u_{s}(0)$ is positive definite and that $u_{s}(0)$ is injective. Then:

Proposition 4. Let $P_{1}^{*}$ be the minimal positive value such that $u_{s}(P)$ ceases to be (positive) definite and $P_{2}^{*}$ the minimal positive value of $P$ such that $u_{s}(P)$ ceases to be injective. Then, $P_{1}^{*} \leq P_{2}^{*}$. Moreover, if there is an Hilbert basis of eigenvectors of $u_{s}(P)$ for all $P<P_{2}^{*}$, then $P_{1}^{*}=P_{2}^{*}$.

P r o of. Let us first recall some results about the spectrum $\mathcal{S}\left(u_{s}(P)\right)$ of a self-adjoint continuous operator. The residual spectrum is empty which means that $\mathcal{S}\left(u_{s}(P)\right)=\mathcal{E}\left(u_{s}(P)\right) \cup \mathcal{S}_{c}\left(u_{s}(P)\right)$ where $\mathcal{E}\left(u_{s}(P)\right)$ is the set of eigenvalues of $u_{s}(P)$ and $\mathcal{S}_{c}\left(u_{s}(P)\right)$ is the continuous spectrum of $u_{s}(P)$. Because $u_{s}(P)$ is a self-adjoint operator, $\mathcal{S}\left(u_{s}(P)\right)$ is a non empty set included in $\mathbb{R}$. Moreover, let us put for all $P$

$$
m(P)=\inf _{x \in \mathcal{H},(x, x)=1}\left(u_{s}(P)(x) \mid x\right) \text { and } M(P)=\sup _{x \in \mathcal{H},(x, x)=1}\left(u_{s}(P)(x) \mid x\right) .
$$

Then $m(P) \in \mathcal{S}\left(u_{s}(P)\right), M(P) \in \mathcal{S}\left(u_{s}(P)\right)$, and $\mathcal{S}\left(u_{s}(P)\right) \subset[m(P), M(P)]$.

If $u_{s}(P)$ is not injective then $u_{s}(P)$ is no longer definite which proves that $P_{1}^{*} \leq P_{2}^{*}$.

Let $0 \leq P<P_{2}^{*}$ and suppose now that there is a Hilbert basis $\left(\xi_{n}(P)\right)_{n \geq 1}$ of eigenvectors associated with a sequence $\mathcal{E}\left(u_{s}(P)\right)=\left(\lambda_{n}(P)\right)_{n \geq 1}$ of eigenvalues with $\lambda_{1}(P)$ the minimum of these eigenvalues. Thus, if $x \in \mathcal{H}$ then $x=\sum_{n=1}^{\infty} x_{n} \xi_{n}(P)$ and $\left(u_{s}(P)(x) \mid x\right)=\sum_{n=1}^{\infty} x_{n}^{2} \lambda_{n}(P)$ which proves that $P_{2}^{*}$ is characterized by $\lambda_{1}\left(P_{2}^{*}\right)=0$. Thus, as long as $0 \leq P<P_{2}^{*},\left(u_{s}(P)(x) \mid x\right) \geq\|x\|^{2} \lambda_{1}\left(P_{2}^{*}\right)>0$ so that $P_{2}^{*} \leq P_{1}^{*}$ and, thanks to the above inequality, $P_{1}^{*}=P_{2}^{*}$.

Note that there is a big difference with the finite dimension case where the spectrum is equal to the set of eigenvalues and where the spectral theorem guarantes the existence of a Hilbert basis of eigenvectors. For compact symmetric (or self adjoint because the spaces are on $\mathbb{R}$ ) operators, the spectral theorem allows to extend naturally this result. There is (see [44] for example) a generalized spectral theorem for self-adjoint (not necessarily compact) which uses the concept of spectral family $\left(E_{\lambda}(P)\right)_{\lambda \in \mathbb{R}}$ associated to $u_{s}(P)$ we seek to avoid. 
The plane to answer issues 2 and 3 is then the following. From Theorem 2, the optimal solution of the ki.s.s. issue is then the minimal positive value $P_{1}^{*}$ such that $u_{s}\left(P_{1}^{*}\right)$ ceases to be definite positive. In other words, with above notations, it is the minimal value $P_{1}^{*}$ such that $m\left(P_{1}^{*}\right)=0$. It is very challenging to evaluate $P_{1}^{*}$ whereas the calculation of $P_{2}^{*}$ remains reasonable. In the following section, we then first calculate the operator $u_{s}(P)$ for the Beck column namely for $u=\tilde{A}$. In a second time, we calculate the critical value $P_{2}^{*}$ for $\tilde{A}_{s}(P)$. In a third step, we compute the associated destabilizing kinematic constraint $c^{*}\left(P_{2}^{*}\right)$. The issue $P_{1}^{*}=P_{2}^{*}$ is reported to the Sect. 4.

\section{Solution for the kernel of the operators $A_{s}(P)$ and $\tilde{A}_{s}(P)$}

In this section, we find the minimal positive value $P_{2}^{*}$ such that $A_{s}\left(P_{2}^{*}\right)$ ceases to be injective. Obviously $\phi\left(P_{2}^{*}\right)$ will then be no more definite positive and the second order work criterion will fail as expected.

The approach is the following. Firstly, we compute the operator $A_{s}(P)$ associated to the symmetric part $\phi_{s}(P)$ of $\phi(P)$. $A_{s}(P)$ could be formally thought as the symmetric part of $A(P)$. In a second step, we compute the kernel of $A_{s}(P)$ and then the value $P_{2}^{*}$ that is the minimal positive value making $A_{s}(P)$ not injective. However, we have to keep in mind that $A(P)$ is defined via the functional (differential) equation (1) but the computation of $A_{s}$ from $A$ is not direct. As mentioned above we will start from the variational formulation. We then will deduce the expression of $A_{s}$ and the subspace $\mathcal{U}_{s}$ associated with $A_{s}$ from the weak formulation. $\mathcal{U}_{s}$ is different of $\mathcal{U}$ and its characterization will naturally emerge from the calculations. That means that, formally, $A_{s}=\frac{1}{2}\left(A+A^{*}\right)$ where $A^{*}$ is the adjoint of $A$. But $A^{*}$ is itself defined for another third subspace $\left(\mathcal{U}_{*}\right)$ of $\mathcal{V}$ different from $\mathcal{U}$ and from $\mathcal{U}_{s}$. The use of the adjoint operator is actually not necessary in our problem. As already mentioned in the introduction, one may find, in the same framework of nonconservative systems, other computations that use the adjoint of an operator, for example in $[13,34,37]$ but in a totally different goal. Indeed, these authors used the so-called adjoint system in order to better approximate the solution of the dynamic equations (namely with the inertial terms) and to investigate the flutter instability of nonconservative systems. The so-called adjoint system in these papers is actually nothing else than the system whose differential operator (namely for the strong formulation) is the adjoint $A^{*}(P)$ of the initial $A(P)$. In fact, we may and we will bypass the use of the adjoint operator. It is however interesting to note that, in [13], concrete mechanical systems associated with $A^{*}$ and $2 A_{s}$ are proposed. Describing mechanical systems associated with these operators is, in general, a challenge even if, for the Beck columns, it is possible. For the ki.s.s. issue, it is moreover not significant. In fact, the way followed in $[13,34,37]$ used the scalar product $\left(. \mid\right.$.) of $\mathcal{L}^{2}([0, \ell])$ which induced topological difficulties for defining the adjoint and the symmetric part of the operator $A(P)$. Using the more appropriate scalar product (.|.) $)_{, 2}$ on $\mathcal{V}$ leads to $\tilde{A}$ and $\tilde{A}^{*}$ which are both elements of $\mathcal{L}_{c}(\mathcal{V})$. There is then no difficulty to define $\tilde{A}_{s}=\frac{1}{2}\left(\tilde{A}+\tilde{A}^{*}\right)$. However, even if the topological difficulties are removed, we now directly calculate $A_{s}(P)$ and $\tilde{A}_{s}(P)$ without calculating $\tilde{A}^{*}$.

\subsection{Calculation of $A_{S}(P)$}

We then start from the variational formulation namely from the bilinear map $\phi=\phi(P)$ on $\mathcal{V}$ defined by (5) or (6). Symmetrizing $\phi$ leads to:

$$
\begin{aligned}
\phi_{s}(w, v) & =\phi_{s}(P)(w, v)=\frac{1}{2}(\phi(P)(w, v)+\phi(P)(v, w)) \\
& =\int_{0}^{\ell}\left(E I w^{\prime \prime}(x) v^{\prime \prime}(x)+\frac{1}{2} P\left(w^{\prime \prime}(x) v(x)+w(x) v^{\prime \prime}(x)\right) d x \quad \forall w, v \in \mathcal{V}\right. \\
& =\int_{0}^{\ell}\left(E I w^{\prime \prime}(x) v^{\prime \prime}(x)+\frac{1}{2} P\left(w^{\prime \prime}(x) v(x)+v^{\prime \prime}(x) w(x)\right) d x \quad \forall w, v \in \mathcal{V}\right.
\end{aligned}
$$

Thus, by definition, $\mathcal{U}_{s}$ is a (dense) subspace of $\mathcal{V}$ such that $v \rightarrow \phi_{s}(P)(w, v)$ is continuous for $\|$.$\| . If w \in \mathcal{U}_{s}$, then by the Riez theorem, there is a vector $A_{s}(P)(w)$ in $\mathcal{L}^{2}([0, \ell])$ such that

$$
\left.\left(A_{s}(P) w\right) \mid j(v)\right)=\phi_{s}(P)(w, v) \quad \forall w, v \in \mathcal{V} .
$$

We are now interested in getting concretely $\mathcal{U}_{s}$ and $A_{s}(P)$ for the Beck column. Then:

Proposition 5. With the above notations and if $\mathcal{H}^{4}(0, \ell)=\left\{v \in L^{2}(0, \ell) \mid v^{\prime \prime \prime \prime} \in L^{2}(0, \ell)\right\}$, then:

$$
\mathcal{U}_{s}=\left\{w \in \mathcal{H}^{4}(0, \ell) \cap \mathcal{V} \mid \begin{array}{r}
\frac{P}{2} w(\ell)+E I w^{\prime \prime}(\ell)=0 \\
\frac{P}{2} w^{\prime}(\ell)+E I w^{(3)}(\ell)=0
\end{array}\right\}
$$


and

$$
\left.A_{s}(P)(w)(x)=E I w^{(4)}(x)+P w^{\prime \prime}(x) \quad \forall x \in\right] 0, \ell\left[\forall w \in \mathcal{U}_{s}\right.
$$

Pr o o f. In order to get concretely $\mathcal{U}_{s}$ and $A_{s}(P)$, we look for regular functions.

Let be $w \in \mathcal{H}^{4}(0, \ell) \cap \mathcal{V}$ and $v \in \mathcal{V}$. Integrating by parts two times in (24) while keeping only the function $v$ and not its derivatives in the integral leads to (without forgetting that $w(0)=w^{\prime}(0)=v(0)=v^{\prime}(0)=0$ because $v$ and $w$ belong to $\mathcal{V}$ ):

$$
\begin{aligned}
\phi_{s}(w, v)= & \int_{0}^{\ell}\left(E I w^{\prime \prime}(x) v^{\prime \prime}(x)+\frac{1}{2} P\left(w^{\prime \prime}(x) v(x)+w(x) v^{\prime \prime}(x)\right) d x\right. \\
= & \int_{0}^{\ell}\left(E I w^{(4)}(x)+P w^{\prime \prime}(x)\right) v(x) d x+\frac{P}{2}\left(w(\ell) v^{\prime}(\ell)-w^{\prime}(\ell) v(\ell)\right) \\
& +E I w^{\prime \prime}(\ell) v^{\prime}(\ell)-E I w^{(3)}(\ell) v(\ell) \\
= & \int_{0}^{\ell}\left(E I w^{(4)}(x)+P w^{\prime \prime}(x)\right) v(x) d x \\
& +\left(E I w^{\prime \prime}(\ell)+\frac{P}{2} w(\ell)\right) v^{\prime}(\ell)-\left(E I w^{(3)}(\ell)+\frac{P}{2} w^{\prime}(\ell)\right) v(\ell) .
\end{aligned}
$$

Using, as usual, functions $v$ with compact support in $] 0, \ell[$, we first deduce that

$$
\left.A_{s}(P)(w)(x)=E I w^{\prime \prime \prime \prime}(x)+P w^{\prime \prime}(x) \forall x \in\right] 0, \ell[
$$

In a second time, relations $(26)+(29)$ lead to:

$$
\begin{aligned}
& \frac{P}{2} w(\ell)+E I w^{\prime \prime}(\ell)=0, \\
& \frac{P}{2} w^{\prime}(\ell)+E I w^{\prime \prime \prime}(\ell)=0 .
\end{aligned}
$$

\subsection{Calculation of $\tilde{A}_{s}(P)$}

Proposition 6. With the above notations, for all $w \in \mathcal{V}$

$$
\tilde{A}_{s}(P)(w)(x)=E I w(x)+P \int_{0}^{x}\left(\int_{0}^{t}\left(w(s)+\frac{1}{2}\left((\ell-s) w^{\prime}(\ell)-w(\ell)\right)\right) d s\right) d t
$$

Pr o of . Calculations similar to those used for $\tilde{A}(P)$.

We may deduce the following

\section{Proposition 7.}

$$
\operatorname{ker} A_{s}(P)=\operatorname{ker} \tilde{A}_{s}(P)=\left\{\begin{aligned}
E I w^{(4)}(x)+P w^{\prime \prime}(x) & =0 \quad \forall x \in] 0, \ell[ \\
\frac{P}{2} w(\ell)+E I w^{\prime \prime}(\ell) & =0 \\
w \in \mathcal{C}^{\infty}\left([0, \ell] \mid \frac{P}{2} w^{\prime}(\ell)+E I w^{(3)}(\ell)\right. & =0 \\
w(0) & =0 \\
w^{\prime}(0) & =0
\end{aligned}\right\}
$$

Pr o o f. Calculations and reasoning are strictly similar to those done to prove that $\tilde{A}(P)$ is injective. 


\subsection{Calculation of the critical load $\boldsymbol{P}_{2}^{*}$}

From (33), we deduce that, if $w \in \operatorname{ker} A_{s}(P)=\operatorname{ker} \tilde{A}_{s}(P)$ then there are $a, b, c, d \in \mathbb{R}$ such that:

$$
w(x)=a x+b+c \cos (\omega x)+d \sin (\omega x) \quad \forall x \in] 0, \ell[
$$

where $\omega^{2}=\frac{P}{E I}$.

Reporting (34) in the set of boundary conditions leads to:

$$
\left\{\begin{aligned}
b+c & =0 \\
a+d \omega & =0 \\
\frac{P}{2}(a \ell+b)+\left(\frac{P}{2}-\omega^{2} E I\right)(c \cos \omega \ell+d \sin \omega \ell) & =0 \\
\frac{P}{2} a+\left(\frac{P}{2}-\omega^{2} E I\right) \omega(-c \sin \omega \ell+d \cos \omega \ell) & =0
\end{aligned}\right.
$$

which also reads (thanks to $\omega^{2} E I=P$ or $\frac{P}{2}-\omega^{2} E I=-\frac{P}{2}$ )

$$
\left\{\begin{aligned}
b & =-c, \\
a & =-d \omega, \\
\frac{P}{2}(-d \omega \ell-c)-\frac{P}{2}(c \cos \omega \ell+d \sin \omega \ell) & =0, \\
-d \omega \frac{P}{2}-\omega \frac{P}{2}(-c \sin \omega \ell+d \cos \omega \ell) & =0 .
\end{aligned}\right.
$$

The two last equations read:

$$
\left\{\begin{aligned}
c(1+\cos \omega \ell)-d(\omega \ell+\sin \omega \ell)) & =0, \\
-c \sin \omega \ell+d(1+\cos \omega \ell) & =0 .
\end{aligned}\right.
$$

The existence of a not nil solution is equivalent to the vanishing of the determinant $\Delta(\omega)$ of this system. One finds:

$$
\Delta(\omega)=2+2 \cos \omega \ell+\omega \ell \sin \omega \ell .
$$

The transcendant equation $\Delta(\omega)=0$ is the analogous of the equation

$$
\operatorname{det} K_{s}(P)=k^{2}-\frac{P^{2} \ell^{2}}{16}=0
$$

used for finding the minimal value $\tilde{P}^{*}$ of the load allowing a destabilizing effect by adding one or several well chosen kinematic constraints (see $[7,21,29]$ ) (see also just hereafter)

If $\sigma^{*}$ the lowest positive root of the equation

$$
2+2 \cos \sigma+\sigma \sin \sigma=0
$$

then $P_{2}^{*}=\frac{\sigma^{* 2} E I}{\ell^{2}}$ and $\sigma^{*}=\pi$. Thus:

$$
P_{2}^{*}=\pi^{2} \frac{E I}{\ell^{2}} .
$$

\subsection{Calculation of $\operatorname{ker} \boldsymbol{A}_{s}\left(P_{2}^{*}\right)=\operatorname{ker} \tilde{A}_{s}\left(P_{2}^{*}\right)$}

For the value $\sigma^{*}=\pi$ of $\sigma=\omega \ell$ and up to a multiplicative constant, we find $d^{*}=0, c^{*}=1, b^{*}=-1$, and $a^{*}=0$ and we get the following vector $w^{*}$ of $\operatorname{ker} A_{s}$ :

$$
w^{*}(x)=1-\cos \left(\pi \frac{x}{\ell}\right) .
$$

For the critical value, $\operatorname{ker} A_{s}\left(P_{2}^{*}\right)=\mathbb{R} w^{*}$.

In the finite dimensional case $n=2$ ( 2 d.o.f. Ziegler system) and for the corresponding critical value $\tilde{P}^{*}=4 \frac{k}{\ell}$, $K_{s}\left(\tilde{P}^{*}\right)=\left(\begin{array}{ll}0 & 0 \\ 0 & k\end{array}\right)$, and $\operatorname{ker} K_{S}\left(\tilde{P}^{*}\right)=\mathbb{R} \tilde{X}^{*}=\mathbb{R}\left(\begin{array}{l}1 \\ 0\end{array}\right)$. It is important to underline again that, for finite discrete systems, there is no difference between $P_{1}^{*}$ and $P_{2}^{*}$. It is the reason why it is denoted by $P^{*}$. We are now ready to find the corresponding optimal destabilizing kinematic constraint. 


\subsection{Calculation of the optimal destabilizing constraint}

Put $(w, v) \rightarrow \phi_{a}(P)(w, v)=\frac{1}{2}(\phi(P)(w, v)-\phi(P)(v, w))$ the skew symmetric (bilinear) part of $\phi(P)$ on $\mathcal{V}^{2}$ so that $\phi(P)=\phi_{s}(P)+\phi_{a}(P)$ on $\mathcal{V}^{2}$.

Thus, for the above vector $w^{*} \in \mathcal{V}$

$$
\forall v \in \mathcal{V} \phi(P)\left(w^{*}, v\right)=\phi_{a}(P)\left(w^{*}, v\right) .
$$

We then define the optimal constraint by:

Definition 1. With the above assumptions and notations, "the" optimal constraint $c^{*} \in \mathcal{V}^{\prime}$ (obviously up to a multiplicative constant) is defined by:

$$
c^{*}(v)=\phi_{a}\left(P_{2}^{*}\right)\left(w^{*}, v\right) \quad \forall v \in \mathcal{V} .
$$

Thanks to (40) and to the continuity of $\phi(P), c^{*}$ is a continuous map. (41) is in accordance with (19) with the Lagrange multiplier $\lambda=1$. The necessary condition $\phi_{s}\left(P_{2}^{*}\right)\left(w^{*}, w^{*}\right)=0$ obviously holds and $\phi\left(P_{2}^{*}\right)\left(w^{*}, v\right)=0 \quad \forall v \in F=\operatorname{ker} c^{*}$ which signs the divergence instability of the constrained system.

We now illustrate this reasoning on the Beck column. We then have the

Proposition 8. For the Beck column, the optimal constraint $c^{*}$ has the following equivalent forms:

$$
\begin{aligned}
\forall v \in \mathcal{V} \quad c^{*}(v) & =v^{\prime}(\ell) \\
& =\int_{0}^{\ell} v^{\prime \prime}(x) d x
\end{aligned}
$$

where (43) has the form of (14) with $a_{1}(x)=1, a_{2}(x)=a_{3}(x)=0$ or alternatively the form of $(15)$ with $a: x \rightarrow a(x)=$ $x^{2} \in \mathcal{V}$. For this form of the constraint, the Lagrange multiplier is $-P_{2}^{*}$.

Proof. For all $w, v \in \mathcal{V}, \phi_{a}(P)(w, v)=\frac{P}{2} \int_{0}^{\ell}\left(w^{\prime \prime}(x) v(x)-w(x) v^{\prime \prime}(x)\right) d x$. Integrating by parts and using that $v$ and $w$ are in $\mathcal{V}$, one gets:

$$
\phi_{a}(P)(w, v)=\frac{P}{2}\left(w^{\prime}(\ell) v(\ell)-w(\ell) v^{\prime}(\ell)\right) .
$$

But $w^{*}(\ell)=2$ and $w^{*^{\prime}}(\ell)=0$ so that $\phi_{a}\left(P_{2}^{*}\right)\left(w^{*}, v\right)=-P_{2}^{*} v^{\prime}(\ell)$.

It is interesting to compare the solution of the destabilizing constraint of the Beck column with the destabilizing constraint $\theta_{2}=0$ of the 2 d.o.f. Ziegler system (see Sect. (1.3)). Two ways to generalize such a relation could be imagine. The first one should be the direct generalization of any linear form $\Theta=\left(\theta_{1}, \theta_{2}\right) \rightarrow C(\Theta)=\alpha_{1} \theta_{1}+\alpha_{2} \theta_{2}$. Because of the relation $v^{\prime}=\theta$ of the Euler-Bernouilli model, we could think to a linear form $v \rightarrow \int_{0}^{\ell} \alpha(x) v^{\prime}(x) d x$. In fact, the solution has not this form for any function $\alpha \in \mathcal{L}^{2}([0, \ell])$ but only for a Dirac distribution at the end $x=\ell$ of the beam. The second idea should be to imagine a discrete Ziegler system with an increasing degree of freedom $n \rightarrow \infty$ by considering the system as an assembly of $n$ successive bars with length $\frac{\ell}{n}$. The constraint, that destabilizes such a discrete system, reads $C_{n}^{*}(\Theta)=\theta_{n}=0$. Thus, for $n \rightarrow \infty, C_{n}^{*}$ approaches the constraint $c^{*}$. We may also remark that, as for the Ziegler systems, the optimal kinematic constraint makes the constrained system a conservative system. It is especially clear from boundaries conditions (2) and (4): $w^{\prime}(\ell)=0$ is the suitable condition so that these boundaries conditions are the same. We however stress that it is not general: the optimal constraint does not necessarily make the constrained system a conservative one.

$$
4 P_{1}^{*}=P_{2}^{*}
$$

The above calculations give the optimal solution with respect to the value $P_{2}^{*}$ namely with respect to the loss of injectivity of $\tilde{A}_{s}(P)$. In Proposition 4, we established that $P_{2}^{*} \geq P_{1}^{*}$ (with equality in finite dimension). Proposition 4 also showed that it is sufficient to exhibit a Hilbert basis of eigenvectors of $\tilde{A}_{s}$. We then tackle the reciprocal inequality issue .

We can obviously restrict our domain to $\left[0, P_{2}^{*}[\right.$. Thus, we suppose that, for the investigated values of the parameter $P$, $\tilde{A}_{s}(P)$ is symmetric positive and injective so that any eigenvalue $\lambda$ is strictly positive. Moreover, from (32), we deduce that

$$
\tilde{A}_{s}(P)=E I \operatorname{id} \mathcal{V}+P K_{g, s}
$$


with

$$
\begin{aligned}
K_{g, s}(w)(x) & =\int_{0}^{x}\left(\int_{0}^{t}\left(w\left(t_{1}\right)+\frac{1}{2}\left(\left(\ell-t_{1}\right) w^{\prime}(\ell)-w(\ell)\right)\right) d t_{1}\right) d t \quad \forall x \in[0, \ell] \\
& =\int_{0}^{x}\left(\int_{0}^{t} w\left(t_{1}\right) d t_{1}\right) d t+\frac{x^{2}}{4}\left(\ell-\frac{x}{3}\right) w^{\prime}(\ell)-\frac{x^{2}}{2} w(\ell) \forall x \in[0, \ell] .
\end{aligned}
$$

From (44) and because of the term $E I \mathrm{Iid}_{\mathcal{V}}$ which is a homothety, there is no chance for $\tilde{A}_{s}(P)$ to be compact. However, any eigenvector of $K_{g, s}$ remains an eigenvector of $\tilde{A}_{s}(P)$. The initial problem is then brought back to the same problem but for $K_{g, s}$. Note that the decomposition (44) of $\tilde{A}_{s}(P)$ is similar to the well-known decomposition $K=K_{e}+P K_{g}$ of any stiffness matrix (for discrete systems) by a sum of a symmetric elastic part $K_{e}$ and the so-called geometric part $K_{g} . K_{g}$ may be not symmetric when the system is subjected to non conservative loads. Obviously, here, $K_{g}$ has been symmetrized and it is called $K_{g, s} . K_{g, s}$ is an integral operator without kernel. It means that there is no function $N:(x, t) \mapsto N(x, t) \in \mathcal{L}^{2}\left([0, \ell]^{2}\right)$ such that $K_{g, s}(w)(x)=\int_{0}^{\ell}\left(\int_{0}^{\ell} N(x, t) w(t) d t\right.$ for all $w \in \mathcal{V}$ : such a "function" should have to contain a Dirac function at $\ell$. However,

Proposition 9. $K_{g, s}$ is a compact operator of $\mathcal{V}$.

Pr o o f. A straightforward calculation shows that:

$$
\max _{w \in \mathcal{V}}\left|w^{\prime}(x)\right| \leq \sqrt{\ell}\|w\|_{\mathcal{V}, 2}, \quad \max _{w \in \mathcal{V}}|w(x)| \leq \frac{2 \ell \sqrt{\ell}}{3}\|w\|_{\mathcal{V}, 2} .
$$

Let $w$ be any element of $\mathcal{V}$ and $h=K_{g, s}(w)$. Then, using (47) and (46):

$$
\begin{aligned}
\forall x \in[0, \ell], \quad & \left|h^{\prime \prime}(x)\right| \leq \frac{11 \ell \sqrt{\ell}}{6}\|w\|_{\mathcal{V}, 2}, \\
\forall x, y \in[0, \ell], \quad & \left|h^{\prime \prime}(x)-h^{\prime \prime}(y)\right| \leq \frac{2 \sqrt{\ell}}{3}|| w \|_{\mathcal{V}, 2}|x-y| .
\end{aligned}
$$

By the Arzela -Ascoly theorem, any sequence $\left(h_{n}\right)$ in $K_{g, s}(B)$ has a subsequence $\left(h_{\phi(n)}\right)$ such that $\left(h_{\phi(n)}^{\prime \prime}\right)$ converges uniformly on $[0, \ell]$ towards some continuous function. That implies that $\left(h_{\phi(n)}\right)$ converges in $\mathcal{V}$ which exactly means that $K_{g, s}(B)$ is relatively compact.

We now solve the whole eigenvalue problem for $\tilde{A}_{s}(P)$. It has been above solved only for the eigenvalue 0 . We now recall this issue:

Find all the $(\lambda(P), w(P)) \in\left(\mathbb{R}_{+}^{*} \times \mathcal{V}\right)$ such that $\tilde{A}_{s}(P)(w(P))=\lambda(P) w(P)$

which is from now on called the EP (for eigenvalue problem). As mentioned above, we have to solve this question only for $P \in\left[0, P_{2}^{*}=\pi^{2} \frac{E I}{\ell^{2}}\right.$ [ which guarantees that there is no eigenvalue in $\mathbb{R}_{-}$. The above expression (32) of $\tilde{A}_{s}$ shows that any solution $(\lambda(P), w(P))$ of EP is such that (dependency on $P$ is omitted) $w \in \mathcal{C}^{\infty}([0, \ell])$ and:

$$
\left.E I w^{(4)}(x)+P w^{\prime \prime}(x)=\lambda w^{(4)}(x) \quad \forall x \in\right] 0, \ell[, \quad \lambda>0
$$

with the boundary conditions

$$
\left\{\begin{aligned}
w(0) & =0, \\
w^{\prime}(0) & =0, \\
\frac{P}{2} w(\ell)+E I w^{\prime \prime}(\ell) & =\lambda w^{\prime \prime}(\ell), \quad \lambda>0, \\
\frac{P}{2} w^{\prime}(\ell)+E I w^{(3)}(\ell) & =\lambda w^{(3)}(\ell), \quad \lambda>0 .
\end{aligned}\right.
$$

In Appendix 6.2, the calculations are done and allow to exhibit a sequence $\left(\left(\lambda_{n}(P)\right)_{n \geq 0},\left(w_{n}(P)\right)_{n \geq 0}\right)$. Because of the structure of $\tilde{A}_{s}$ given by (44), we deduce from Proposition 9 that:

Proposition 10. For $P<P_{2}^{*}$, the solution of EP is a sequence of $\left(\left(\lambda_{n}(P)\right)_{n \geq 0},\left(w_{n}(P)\right)_{n \geq 0}\right)$, and the family $\left(w_{n}(P)\right)_{n \geq 0}$ is an orthogonal Hilbert basis.

Corollary 11. $P_{1}^{*}=P_{2}^{*}$ and the solution expanded above is the optimal one in the whole.

P r o o f. It directly results from Propositions 4 and 10. 


\section{Stability issues}

As it was clearly claimed at the beginning of this manuscript, only linear divergence-type stability is investigated which means that mass effects are not taken into account. Nevertheless, investigating more accurately links with linear dynamic stability is an interesting challenge. For both finite (Ziegler column) or infinite (Beck column) unconstrained mechanical systems subjected to a complete follower force, no linear divergence-type instability may occur and only linear flutter-type dynamic instability occurs. For example, for a 2 d.o.f. Ziegler system, the flutter-type instability occurs for the value $p_{f}^{*} \approx 2,54$ whereas for the Beck column it occurs for $p_{f}^{*} \approx 20.05$ with dimensionless load parameter $p$. The instability for $p>p_{f}^{*} \approx 20.05$ may be found in 1952 Beck paper [3] whereas the stability for $p<p_{f}^{*} \approx 20.05$ has been proved by Carr and Malhardeen in [5]. Note however that the proof in [5] does not use a direct Lyapounov method.

Here obviously we do not pretend a priori that the constrained system is stable for $P<P^{*}$ but that it is divergence stable for $P<P^{*}$ and that is divergence unstable for $P=P^{*}$. Nevertheless, thanks to a special property of $c^{*}$, the constrained Beck column will turn out to be stable (flutter-type and divergence-type as well). We stress again that we investigate only linear stability.

Let us start by the 2 d.o.f. Ziegler system. Its (linear) dynamic equation reads

$$
M \ddot{X}+K(P) X=0 .
$$

$M$ is the mass matrix. It depends on the mass distribution and is symmetric positive definite. $K(P)$ is given by (3). When the system is subjected to the kinematic constraint $C$, then the dynamic equation of the constrained system reads:

$$
\left\{\begin{aligned}
M \ddot{X}+K(P) X+\lambda C & =0, \\
C^{T} X & =0 .
\end{aligned}\right.
$$

The optimal constraint $C^{*}$ is given by $C^{*}=K\left(P^{*}\right) X^{*}$ with $X^{*} \neq 0 \in \operatorname{ker} K_{s}\left(\tilde{P}^{*}\right) \neq\{0\}$ because $K_{s}\left(\tilde{P}^{*}\right)$ is degenerated (see Sect. 1.3). Multiplying by the left by $K_{S}\left(P^{*}\right) K^{-1}\left(P^{*}\right)$ removes the Lagrange multiplier and (51) then becomes:

$$
M^{*} \ddot{X}+K_{S}\left(\tilde{P}^{*}\right) X=0
$$

with $M^{*}=K_{s}\left(P^{*}\right) K^{-1}\left(P^{*}\right) M$. It is then easy to check that $t \mapsto U(t)=t X^{*}$ is an unbounded solution of (52). It is also interesting to check that $U$ is an unbounded solution of (51) with the Lagrange multiplier $\lambda(t)=-t$ at each time $t$ which proves the linear dynamic instability by divergence of the corresponding equilibrium of the constrained system. Indeed, (51) also reads

$$
\left\{\begin{aligned}
K^{-1}\left(P^{*}\right) M \ddot{X}+X+\lambda X^{*} & =0 \\
\left(X^{*}\right)^{T} K\left(P^{*}\right)^{T} X=\left(X^{*}\right)^{T} K\left(P^{*}\right) X & =0
\end{aligned}\right.
$$

and at each time $t$ :

$$
\left\{\begin{array}{r}
K^{-1}\left(P^{*}\right) M \ddot{U}+U+\lambda X^{*}=(t+\lambda(t)) X^{*}=0 \\
\left(X^{*}\right)^{T} K\left(P^{*}\right)^{T} U=t\left(X^{*}\right)^{T} K\left(P^{*}\right)\left(X^{*}\right)=0
\end{array}\right.
$$

with $\lambda(t)=-t \quad \forall t \geq 0$.

However, it is worth noting that the direct Lyapounov method is ineffective here because of the non symmetry of $M^{*}$ or of $K^{-1}\left(P^{*}\right) M$. Another way to keep a symmetric mass matrix for the constrained system might consist in using the compressions of the mass and stiffness matrices as in [26] for example. In this way, the size of the dynamic system is reduced and the compressed mass matrix remains symmetric positive definite. Unfortunately, the compressed stiffness matrix generally remains non symmetric which again prevents of using the direct Lyapounov method.

However, we may extract by a similar way an unbounded solution of the constrained linear dynamic equation of the Beck column:

$$
\left.\rho \frac{\partial^{2} w}{\partial t^{2}}+E I \frac{\partial^{4} w}{\partial x^{4}}+P \frac{\partial^{2} w}{\partial x^{2}}+\lambda c(w)=0 \quad \forall x \in\right] 0, \ell[\forall t \geq 0
$$

with the same above boundary conditions (2) we may rewrite as:

$$
w(0, t)=\frac{\partial w}{\partial x}(0, t)=\frac{\partial^{2} w}{\partial x^{2}}(\ell, t)=\frac{\partial^{3} w}{\partial x^{3}}(\ell, t)=0 \quad \forall t \geq 0
$$

and with the linear constraint $c(w)=0$ where $c$ is a linear functional.

Suppose now that $P=P_{1}^{*}=P_{2}^{*}=P^{*}$ and that $c=c^{*}$ is built as above: $c^{*}(w)=w^{\prime}(\ell)$. Let $(t, x) \mapsto t u(x)=t(1-$ $\left.\cos \left(\pi \frac{x}{\ell}\right)+\frac{\pi^{2}}{2 \ell^{2}} x^{2}\right)$ be a displacement field suggested by the function $w^{*} \in \operatorname{ker} A_{s}\left(P^{*}\right)$ given by (39). The reader may readily check that $u$ satisfies the boundary conditions (56) (whereas $t w^{*}(x)$ does not satisfy (56)!!). 
However,

$$
\begin{aligned}
\rho \frac{\partial^{2} u}{\partial t^{2}}+E I \frac{\partial^{4} u}{\partial x^{4}}+P^{*} \frac{\partial^{2} u}{\partial x^{2}}+\lambda c^{*}(u) & =t\left(-E I \frac{\pi^{4}}{\ell^{4}} \cos \left(\pi \frac{x}{\ell}\right)+P^{*} \frac{\pi^{2}}{\ell^{2}} \cos \left(\pi \frac{x}{\ell}\right)+\frac{\pi^{2}}{\ell}\right)+\lambda(t) \frac{\pi^{2}}{\ell} \\
& =t \frac{\pi^{2}}{\ell}+\lambda(t) \frac{\pi^{2}}{\ell} \\
& =(t+\lambda(t)) \frac{\pi^{2}}{\ell} \\
& =0
\end{aligned}
$$

for the value $\lambda(t)=-t$ of the Lagrange multiplier and because of the critical value of the loading $P^{*}=P_{2}^{*}=\pi^{2} \frac{E I}{\ell^{2}}$ given by (38). That proves the linear dynamic divergence instability of the constrained system by $c^{*}$ for $P^{*}=P_{2}^{*}=\pi^{2} \frac{E I}{\ell^{2}}$. But, the constraint $c^{*}$ surprisingly leads to a conservative constrained system equivalent to the system $(1)+(4)$ with $P_{c}=P^{*}$ constrained by $c^{*}$. The stability of such a system is easier to study because only divergence-type instabilities may occur. It follows that the constrained system is stable (both divergence and flutter) if $P<P^{*}=\pi^{2} \frac{E I}{\ell^{2}}$ obviously in the linear framework. Besides, an energy functional $\mathcal{E}$ may be built for this constrained system:

$$
\mathcal{E}(w)=\frac{1}{2}\left(\int_{0}^{\ell} \rho\left(\frac{\partial w}{\partial t}\right)^{2} d x\right)+\frac{1}{2} \phi_{s}(P)(w, w) \quad \forall w \in F=\operatorname{ker} c^{*}
$$

To conclude we may remark that contrary to the finite dimensional case and because of the boundary conditions, we cannot choose exactly $u(t, x)=t w^{*}(x)$ with $w \in \operatorname{ker} A_{s}\left(P^{*}\right)$ but we have to introduce a corrective term $t \frac{\pi^{2}}{2 \ell^{2}} x^{2}$ to build a non bounded solution of dynamic equations. We also may observe that $P^{*}=\pi^{2} \frac{E I}{\ell^{2}}<20.5 \frac{E I}{\ell^{2}}$. However, no general stability analysis of any constrained Beck column has been done which should lead to a general flutter KISS investigation and which is out of the scope of this paper. A general flutter KISS investigation has been led for 3 d.o.f. Ziegler system in [27]. Finally, the fact that $c^{*}$ leads to a conservative system is a pure chance and is related to the so-called Geometric Degree of Nonconservativity (GDNC) which has been deeply investigated in [25] for linear systems and in [28] for non linear systems as well but only in the discrete mechanic framework.

\section{Conclusion}

In this paper, we propose a direct extension of the divergence ki.s.s. issue to continuum mechanics. To illustrate the effectiveness of the method, we develop the general machinery on the well-known Beck column. We then prove that, as long as the follower force $P$ is lower than $P^{*}=\pi^{2} \frac{E I}{\ell^{2}}$, there is no kinematic constraint that may destabilize this system by divergence. For $P=P^{*}$, the kinematic constraint defined by the linear continuous form $c: v \rightarrow c(v)=v^{\prime}(\ell)$ makes the corresponding mechanical system divergence unstable. To perform this extension, we systematically reason with the variational formulation of the problem involving the bilinear form $\phi_{S}(P)$ and its associated integral operator $\tilde{A}_{s}(P)$. This is a suitable way to progress, to effectively make the calculations and finally to find the critical load $P^{*}$ and the corresponding element $w^{*} \in \operatorname{ker} \tilde{A}_{s}\left(P^{*}\right)$ as well. Because of the infinite dimensional framework, a special attention is paid to topological aspects. These aspects are essential especially for the comparison between the loss of injectivity and the loss of definite positiveness. Contrarily to common situations in mechanics, we cannot use here the compactness of the injection $j: \mathcal{V} \rightarrow \mathcal{L}^{2}([0, \ell])$. Thus, we directly prove that the family of eigenvectors of the involved operator $\tilde{A}_{s}$ is a complete set. Obviously, the same general machinery may be used in any other situation involving nonconservative actions. However, no general result may be invoked to show that the critical values $P_{1}^{*}$ (loss of definite positivity) and $P_{2}^{*}$ (loss of one-to-one property) are equal. This issue must then be accurately and systematically by hands investigated. A last section is devoted to linear dynamic equations. We show that the built solution leads to a unbounded solution $(t, x) \mapsto u(t, x)$ of the dynamic equations of the constrained Beck column for $P=P^{*}$. We also show that the constrained system is both divergence-type and flutter-type linear stable for $P<P^{*}$.

\section{Appendices}

\subsection{Equivalence between different forms of kinematic constraints}

In this appendix we prove the equivalence of the both forms (15) and (14) of any kinematic constraint $c \in \mathcal{V}^{\prime}$. First, because $a^{\prime \prime} \in \mathcal{L}^{2}([0, \ell])$ if $a \in \mathcal{V}$, it is obvious that (15) is a particular case of (14) with $a_{1}=a^{\prime \prime}, a_{2}=0, a_{3}=0$. Reciprocally, 
by linearity, it is sufficient to show apart that $\int_{0}^{\ell} a_{1}(x) v^{\prime \prime}(x) d x, \int_{0}^{\ell} a_{2}(x) v^{\prime}(x) d x$ and $\int_{0}^{\ell} a_{3}(x) v(x) d x$ can be written as $\int_{0}^{\ell} a^{\prime \prime}(x) v^{\prime \prime}(x) d x$ with $a$ an element of $\mathcal{V}$. We do it by two steps. First, we show that the two last types of integrals $\int_{0}^{\ell} a_{2}(x) v^{\prime}(x) d x$ and $\int_{0}^{\ell} a_{3}(x) v(x) d x$ may be written with the same form as the first one namely $\int_{0}^{\ell} a_{1}(x) v^{\prime \prime}(x) d x$ and we do it only explicitly for the first one $\int_{0}^{\ell} a_{2}(x) v^{\prime}(x) d x$. We omit to justify the validity of the derivatives and integrals. The fact that the interval is bounded is fundamental. In the second step, we show that any $\int_{0}^{\ell} a_{1}(x) v^{\prime \prime}(x) d x$ has the form of (15) which achieves the proof.

Step 1

Let $\int_{0}^{\ell} a_{2}(x) v^{\prime}(x) d x$ be any integral of this form. Put $a_{1}(x)=-\int_{x}^{\ell} a_{2}(t) d t$. Then $a_{1}^{\prime}(x)=-a_{2}(x)$ and $a_{1}(\ell)=0$. After integrating by parts:

$$
\int_{0}^{\ell} a_{2}(x) v^{\prime}(x) d x=\left[-a_{1}(x) v^{\prime}(x)\right]_{0}^{\ell}-\int_{0}^{\ell}\left(-a_{1}(x)\right) v^{\prime \prime}(x) d x=\int_{0}^{\ell} a_{1}(x) v^{\prime \prime}(x)
$$

since $a_{1}(\ell)=0$ et $v^{\prime}(0)=0$. For an integral like $\int_{0}^{\ell} a_{3}(x) v(x) d x$, we have to put $a_{1}(x)=\int_{x}^{\ell}\left(\int_{x}^{\ell} a_{3}(u) d u\right) d t$.

Step 2

Let now $\int_{0}^{\ell} a_{1}(x) v^{\prime \prime}(x) d x$ be any integral of the considered form with $a_{1} \in \mathcal{L}^{2}([0, \ell])$. Put $a(x)=\int_{0}^{x}\left(\int_{0}^{t} a_{1}(u) d u\right) d t$. Then $a$ is two times differentiable and $a(0)=0, a^{\prime}(x)=\int_{0}^{x} a_{1}(u) d u, a^{\prime}(0)=0$ and $a^{\prime \prime}(x)=a_{1}(x)$ (almost everywhere on $[0, \ell])$. We deduce that $\int_{0}^{\ell} a_{1}(x) v^{\prime \prime}(x) d x$ has the appropriate form $\int_{0}^{\ell} a^{\prime \prime}(x) v^{\prime \prime}(x) d x$ with $a(0)=a^{\prime}(0)=0$ namely that $a \in \mathcal{V}$.

\subsection{Eigenvalues problem for $\tilde{A}_{s}(P)$}

In this appendix, we solve EP given by (48) and (49). (48) also reads:

$$
\left.(E I-\lambda) w^{(4)}(x)+P w^{\prime \prime}(x)=0 \quad \forall x \in\right] 0, \ell[, \quad \lambda>0 .
$$

If $\lambda=E I$ and since $P \neq 0$, then $w^{\prime \prime}(x)=0$ and because $w \in \mathcal{V}$, the natural boundary conditions $w(0)=w^{\prime}(0)=0$ leads to $w=0$. Thus $\lambda \neq 0$. Two different cases may occur: $\lambda<E I$ and $\lambda>E I$.

\subsection{1 $\left.\mathcal{E}\left(\tilde{\boldsymbol{A}}_{s}(\boldsymbol{P})\right) \cap\right] \mathbf{0 ,} \boldsymbol{E I}[$}

Put here $\omega^{2}=\frac{P}{E I-\lambda}$ namely $\lambda=E I-\frac{P}{\omega^{2}}$ and, because $\lambda>0$, then $\omega^{2}>\frac{P}{E I}$. Then the solution $w$ has the following form:

$$
w(x)=a x+b+c \cos (\omega x)+d \sin (\omega x) \quad \forall x \in] 0, \ell[.
$$

The two first boundary conditions of (49) lead to $b=-c$ and $a=-d \omega$ which gives

$$
w(x)=-d \omega x-c+c \cos (\omega x)+d \sin (\omega x) \quad \forall x \in] 0, \ell[.
$$

We deduce successively

$$
\left.w^{\prime}(x)=-d \omega-c \omega \sin (\omega x)+d \omega \cos (\omega x) \quad \forall x \in\right] 0, \ell[
$$

and

$$
\left.w^{\prime \prime}(x)=-c \omega^{2} \cos (\omega x)-d \omega^{2} \sin (\omega x) \quad \forall x \in\right] 0, \ell[
$$

and lastly

$$
\left.w^{\prime \prime \prime}(x)=c \omega^{3} \sin (\omega x)-d \omega^{3} \cos (\omega x) \quad \forall x \in\right] 0, \ell[.
$$

Thus, the two last boundary conditions of (49) read:

$$
\begin{aligned}
\frac{P}{2} w(\ell)+\frac{P}{\omega^{2}} w^{\prime \prime}(\ell) & =0, \\
\frac{P}{2} w^{\prime}(\ell)+\frac{P}{\omega^{2}} w^{(3)}(\ell) & =0
\end{aligned}
$$


or

$$
\begin{gathered}
\frac{1}{2}(-d \omega \ell-c+c \cos (\omega \ell)+d \sin (\omega \ell))+\frac{1}{\omega^{2}}\left(-c \omega^{2} \cos (\omega \ell)-d \omega^{2} \sin (\omega \ell)\right)=0, \\
\frac{1}{2}(-d \omega-c \omega \sin (\omega \ell)+d \omega \cos (\omega \ell))+\frac{1}{\omega^{2}}\left(c \omega^{3} \sin (\omega \ell)-d \omega^{3} \cos (\omega \ell)\right)=0
\end{gathered}
$$

or again

$$
\begin{aligned}
\frac{1}{2}(-d \omega \ell-c+c \cos (\omega \ell)+d \sin (\omega \ell))-c \cos (\omega \ell)-d \sin (\omega \ell) & =0 \\
\left.\frac{1}{2}(-d-c \sin (\omega \ell)+d \cos (\omega \ell))+c \sin (\omega \ell)-d \cos (\omega \ell)\right) & =0 .
\end{aligned}
$$

Thus, $(c, d)$ must be a non trivial solution of

$$
\left\{\begin{aligned}
c(\cos (\omega \ell)+1)+d(\omega \ell+\sin (\omega \ell)) & =0 \\
c \sin (\omega \ell)-d(\cos (\omega \ell)+1) & =0 .
\end{aligned}\right.
$$

Put $\sigma=\omega \ell$. In order to have a non trivial solution, the determinant of (64) must vanish which reads:

$$
\sigma \sin \sigma+2+2 \cos \sigma=0 .
$$

Remark that (64) is not equivalent to (35) whereas (65) is the same equation as (37). Contrary to the computation of $P_{2}^{*}$, we have now to investigate all the solutions $\sigma_{n}$ for $n \geq 1$. The subset of positive solutions of (65) is built by an increasing sequence $\left(\sigma_{n}\right)_{n \geq 1}$ whose $((2 n-1) \pi)_{n \geq 1}$ is a subsequence of solutions. The approximations of the four first solutions are: $\sigma_{1}=\pi, \sigma_{2} \approx 5,596, \sigma_{3}=3 \pi, \sigma_{4} \approx 12,242$. We then have

$$
\sigma_{2 n-1}=(2 n-1) \pi \text { and }(2 n-1) \pi<\sigma_{2 n}<(2 n+1) \pi \forall n \geq 1
$$

The corresponding eigenvalues are $\lambda_{n}=E I-\frac{P \ell^{2}}{\sigma_{n}^{2}}$ for $n \geq 1$ and the corresponding eigenvectors read

$$
\left.w_{2 n}(x)=-\frac{x}{\ell}+\frac{1}{2}-\frac{1}{2} \cos \left(\sigma_{2 n} \frac{x}{\ell}\right)+\frac{1}{\sigma_{2 n}} \sin \left(\sigma_{2 n} \frac{x}{\ell}\right) \quad \forall x \in\right] 0, \ell[\quad \forall n \geq 1
$$

and

$$
\left.w_{2 n-1}(x)=\cos \left((2 n-1) \pi \frac{x}{\ell}\right)-1 \quad \forall x \in\right] 0, \ell[\quad \forall n \geq 1
$$

because, from (65) for $\omega_{n} \ell=(2 n-1) \pi$, we deduce that $d=0$ and $c$ is any.

Remark that, thanks to $P<P_{2}^{*}, \lambda_{n}>0$ for all $n \geq 1$.

$$
\text { 6.2.2 } \left.\mathcal{E}\left(\tilde{\boldsymbol{A}}_{s}(\boldsymbol{P})\right) \cap\right] \boldsymbol{E} \boldsymbol{I},+\infty[
$$

Put here $\omega^{2}=\frac{P}{-E I+\lambda}$ namely $\lambda=E I+\frac{P}{\omega^{2}}$. Then the solution $w$ has the following form:

$$
w(x)=a x+b+c \cosh (\omega x)+d \sinh (\omega x) \quad \forall x \in] 0, \ell[.
$$

The two first boundary conditions of (49) lead to $b=-c$ and $a=-d \omega$ which gives

$$
w(x)=-d \omega x-c+c \cosh (\omega x)+d \sinh (\omega x) \quad \forall x \in] 0, \ell[.
$$

We deduce successively

$$
\left.w^{\prime}(x)=-d \omega+c \omega \sinh (\omega x)+d \omega \cosh (\omega x) \quad \forall x \in\right] 0, \ell[
$$

and

$$
\left.w^{\prime \prime}(x)=c \omega^{2} \cosh (\omega x)+d \omega^{2} \sinh (\omega x) \quad \forall x \in\right] 0, \ell[
$$

and lastly

$$
\left.w^{\prime \prime \prime}(x)=c \omega^{3} \cosh (\omega x)+d \omega^{3} \sinh (\omega x) \quad \forall x \in\right] 0, \ell[
$$


Thus, the two last boundary conditions of (49) read:

$$
\begin{aligned}
\frac{P}{2} w(\ell)-\frac{P}{\omega^{2}} w^{\prime \prime}(\ell) & =0, \\
\frac{P}{2} w^{\prime}(\ell)-\frac{P}{\omega^{2}} w^{(3)}(\ell) & =0,
\end{aligned}
$$

or

$$
\begin{gathered}
\frac{1}{2}(-d \omega \ell-c+c \cosh (\omega \ell)+d \sinh (\omega \ell))-\frac{1}{\omega^{2}}\left(c \omega^{2} \cosh (\omega \ell)+d \omega^{2} \sinh (\omega \ell)\right)=0 \\
\frac{1}{2}(-d \omega+c \omega \sinh (\omega \ell)+d \omega \cosh (\omega \ell))-\frac{1}{\omega^{2}}\left(c \omega^{3} \sinh (\omega \ell)+d \omega^{3} \cosh (\omega \ell)\right)=0
\end{gathered}
$$

or again

$$
\begin{aligned}
\frac{1}{2}(-d \omega \ell-c+c \cosh (\omega \ell)+d \sinh (\omega \ell))-c \cosh (\omega \ell)-d \sinh (\omega \ell) & =0, \\
\left.\frac{1}{2}(-d+c \sinh (\omega \ell)+d \cosh (\omega \ell))-c \sinh (\omega \ell)-d \cosh (\omega \ell)\right) & =0 .
\end{aligned}
$$

Thus, $(c, d)$ must be a non trivial solution of

$$
\left\{\begin{array}{r}
c(\cosh (\omega \ell)+1)+d(\omega \ell+\sinh (\omega \ell))=0, \\
c \sinh (\omega \ell)+d(\cosh (\omega \ell)+1)=0 .
\end{array}\right.
$$

Put $\sigma=\omega \ell$. In order to have a non trivial solution, the determinant of (73) must vanish which reads:

$$
-\sigma \sinh \sigma+2+2 \cosh \sigma=0
$$

which has only one solution $\sigma_{0} \approx 2,399$ in $\mathbb{R}_{+}^{*}$. There is only one corresponding eigenvalue $\lambda_{0}=E I+\frac{P \ell^{2}}{\sigma_{0}^{2}}$ and the corresponding eigenvector is

$$
\left.w_{0}(x)=-\frac{x}{\ell}+\frac{1}{2}-\frac{1}{2} \cosh \left(\sigma_{0} \frac{x}{\ell}\right)+\frac{1}{\sigma_{0}} \sinh \left(\sigma_{0} \frac{x}{\ell}\right) \quad \forall x \in\right] 0, \ell[.
$$

\section{References}

[1] E. Absi and J. Lerbet, Instability of elastic bodies, Mech. Res. Commun. 31, 39-44 (2004).

[2] G. Allaire, Analyse Numérique et Optimisation: Une Introduction à la Modélisation Mathématique (Editions Ecole Polytechnique, 2005).

[3] M. Beck, Die Knicklast des einseitig eingespannten tangential gedrückten Stabes, Z. Angew. Math. Phys. 3, 225-228 (1952).

[4] V. V. Bolotin, Non-Conservative Problems of the Theory of Elastic Stability (Pergamon Press, 1963).

[5] J. Carr and Z. M. Malhardeen, Beck's problem, SIAM J. Appl. Math. 37(2), 261-262 (1979).

[6] N. Challamel, F. Nicot, J. Lerbet, and F. Darve, On the stability of non-conservative elastic systems under mixed perturbations, EJECE 13(3), 347-367 (2009), https://doi.org/10.3166/ejece.13.

[7] N. Challamel, F. Nicot, J. Lerbet, and F. Darve, Stability of non-conservative elastic structures under additional kinematics constraints, Engineering Structures 32, 3086-3092 (2010), pp. 67-84.

[8] E. de Langre and O. Doaré, Edge flutter of long beam under follower loads, JoMMS 10(3), (2015), https://doi.org/ 10.2140/jomms.2015.10.283 msp.

[9] O. Doaré, Dissipation effect on local and global stability of fluid-conveying pipes, J. Sound Vib. 329(1), 72-83 (2010).

[10] O. Doaré, Dissipation effect on local and global fluid-elastic instabilities, in: Nonlinear Physical Systems: Spectral Analysis, Stability and Bifurcations, edited by O. N. Kirillov and D. E. Pelinovsky (Wiley, Hoboken, NJ, 2014), pp. 67-84.

[11] O. Doaré and E. de Langre, Local and global instability of fluid-conveying pipes on elastic foundations, J. Fluid. Struct. 16(1), $1-14$ (2002).

[12] I. Elishakoff, Controversy associated with the so-called "follower forces": critical overview, Appl. Mech. Rev. 58(1-6), 117-142 (2005).

[13] G. Herrmann, Dynamics and Stability of Mechanical Systems with Follower Forces, Monography (Report NASA CR-1782, 1971), p. 234.

[14] R. Hill, Some basic principles in the mechanics of solids without a natural time, J. Mech. Phys. Solids 7, 209-225 (1959).

[15] R. Jurisits and A. Steindl, Mode interactions and resonances of an elastic fluid-conveying tube, PAMM 11(1), 323-324 (2011).

[16] W. T. Koiter, Unrealistic follower forces, J. Sound Vib. 194, 636-638 (1996).

[17] O. N. Kirillov and F. Verhulst, Paradoxes of dissipation-induced destabilization or who opened Withney's umbrella? Z. Angew. Math. Mech. 90(6), 462-488 (2010).

[18] O. N. Kirillov, Nonconservative Stability Problems of Modern Physics (de Gruyter, Berlin, Boston, 2013). 
[19] R. J. Knops and L. E. Payne, Stability in linear elasticity, Int. J. Solids Struct. 4(12), 1233-1242 (1968).

[20] L. P. Lebedev, I. I. Vorovitch, and G. M. L. Gladwell, Functional Analysis. Application in Mechanics and Inverse Problems (Kluwer Academic Publishers, Dordrecht etc., 2003).

[21] J. Lerbet, M. Aldowaji, N. Challamel, F. Nicot, F. Prunier, and F. Darve, P-positive definite matrices and stability of nonconservative systems, Z. Angew. Math. Mech. 92(5), 409-422 (2012).

[22] J. Lerbet, O. Kirillov, M. Al-Dowaji, F. Nicot, N. Challamel, and F. Darve, Additional constraints may soften a non-conservative structural system: buckling and vibration analysis, Int. J. Solids Struct. 50(2), 636-370 (2013).

[23] F. Nicot, J. Lerbet, and F. Darve, Flutter and divergence instabilities of some constrained twodegree-of-freedom systems, Journal of Engineering Mechanics 140(1), 47-52 (2014).

[24] O. N. Kirillov, N. Challamel, F. Darve, J. Lerbet, and F. Nicot, Singular divergence instability thresholds of kinematically constrained circulatory systems, Phys. Lett. A 378(3), 147-152 (2014).

[25] J. Lerbet, M. Aldowaji, N. Challamel, O. N. Kirillov, F. Nicot, and F. Darve, Geometric degree of nonconservativity, Math. and Mech. of Complex Systems 2(2), 123-139 (2014), https://doi.org/10.2140/memocs.2014.2.123.

[26] J. Lerbet, N. Challamel, F. Nicot, and F. Darve, Variational Formulation of Divergence Stability for constrained systems, Appl. Math. Modell. (2015), https://doi.org/10.1016/j.apm.2015.02.052.

[27] J. Lerbet, G. Hello, N. Challamel, F. Nicot, and F. Darve, 3-Dimensional flutter kinematic structural stability nonlinear analysis: Real world applications (2016), https://doi.org/10.1016/j.nonrwa.2015.10.006.

[28] J. Lerbet, N. Challamel, F. Nicot, and F. Darve, Geometric Degree of Nonconservativity: set of solutions for the linear case and extension to the differentiable non linear case, Appl. Math. Modell. (2016), https://doi.org/10.1016/j.apm.2016.01.030.

[29] J. Lerbet, N. Challamel, F. Nicot, and F. Darve, Kinematical Structural Stability, Discrete and Continuous Dynamical Systems Series S (DCDS-S) of American Institute of Mathematical Sciences (AIMS), DCDS-S 9-2 June 2016 special issue (2016).

[30] R. Mennicken and M. Möller, Non-Self-Adjoint Boundary Eigenvalue Problems, North-Holland Mathematics Studies 192 (Elsevier, 2003).

[31] A. A. Movchan, The direct method of Lyapunov in stability problems of elastic systems, PMM-J. Appl. Math. Mech. 23(3), 686-700 (1959).

[32] A. A. Movchan, Stability of processes with respect to two metrics, PMM-J. Appl. Math. Mech. 24(6), 1506-1524 (1960).

[33] N. N. Moiseyev and V. V. Rumyantsev, Dynamic Stability of Bodies Containing Fluid (Springer, 1968).

[34] S. Nemat-Nasser and G. Herrmann, Adjoint systems in nonconservative problems of elastic stability, AIAA J. 4, 2221-2222 (1966).

[35] M. P. Païdoussis, Fluid-Structure Interactions: Slender Structures and Axial Flows I (Academic Press, San Diego, CA, 1998).

[36] M. P. Païdoussis, Fluid-Structure Interactions: Slender Structures and Axial Flows II (Elsevier, San Diego, CA, 2003).

[37] S. N. Prasad and G. Herrmann, The usefulness of adjoint systems in solving nonconservative stability problems of elastic continua, Int. J. Solids Struct. 5(7), 727-735 (1969).

[38] A. Steindl and H. Troger, One and two-parameter bifurcations to divergence and flutter in the three-dimensional motions of a fluid conveying viscoelastic tube with D4-symmetry, Advances in Nonlinear Dynamics: Methods and Applications 8, 161-178 (1995).

[39] A. Steindl and H. Troger, Nonlinear three-dimensional oscillations of elastically constrained fluid conveying viscoelastic tubes with perfect and broken O(2)-symmetry, Nonlinear Dyn. 7(2), 165-193 (1995).

[40] A. Steindl and H. Troger, Heteroclinic cycles in the three-dimensional postbifurcation motion of O(2)-symmetric fluid conveying tubes, Appl. Math. Comput. 78(2-3), 269-277 (1996).

[41] A. Steindl, Hopf-Takens-Bogdanov interaction for a fluid-conveying tube, PAMM 16(1), 293-294 (2016).

[42] J. M. T. Thompson, 'Paradoxical' mechanics under fluid flow, Nature 296(5853), 135-137 (1982).

[43] H. Troger and A. Steindl, Nonlinear Stability and Bifurcation Theory (Springer, 1991).

[44] J. Weidmann, Linear Operators in Hilbert Spaces (Springer-Verlag, 1980).

[45] H. Ziegler, Principles of Structural Stability (Blaisdell Pub. Company, 1968). 